\title{
GIF NATURAL RADIOCARBON MEASUREMENTS IX
}

\section{GEORGETTE DELIBRIAS, MARIE-THÉRESE GUILLIER, and JACQUES LABEYRIE}

Centre des Faibles Radioactivités, Laboratoire mixte CNRS-CEA, 91190 Gif-sur-Yvette, France

The following list includes ${ }^{14} \mathrm{C}$ measurements of geologic samples, the majority of which pertain to sea-level variations, and of archaeologic samples mainly from France, West Africa, and South America. Most of the dates were measured between 1972 and 1973 when installations were not modified. The technique used is described in Radiocarbon, 1972, v 14, p 280-320. Dates were calculated using the ${ }^{14} \mathrm{C}$ half-life of 5568 years; modern standard is 0.95 of the NBS oxalic acid. Reported errors are one standard deviation for 2000 minutes measurements.

\section{ACKNOWLEDGMENTS}

The authors would like to thank Mireille Rousseau and Rolande Kolbach for sample preparation, Michel Jaudon for technical assistance, and especially Vivian Hendy, for assisting with drafting the paper in English.

\section{SAMPLE DESCRIPTIONS}

I. GEOLOGIC SAMPLES

Most samples were dated to study sea-level variations especially during the Holocene on different coasts of the world.

\section{A. France}

Marais de Cessières series, Aisne

Several profiles were studied by pollen analysis and ${ }^{14} \mathrm{C}$ dates in two large acid peat bogs of Cessières marsh $\left(49^{\circ} 33^{\prime} 14^{\prime \prime} \mathrm{N}, 3^{\circ} 31^{\prime} 38^{\prime \prime} \mathrm{E}\right)$, to survey history of this marsh. Samples coll and subm 1969-1974 by F Morand, Inst Geog, Paris.

Gif-1694. Marais de Cessières, 440/110-115 $4.180 \pm 140$

Peat 1.1 to $1.15 \mathrm{~m}$ below surface, Core 440 . Pollen zone, AtlanticSub-boreal-Alnus abundant. Comment: at base of important detrital accumulation corresponding to land clearing.

Gif-1695. Marais de Cessières, 040/105-110 $1300 \pm 100$

Peat 1.05 to $1.1 \mathrm{~m}$ below surface, Core 040 . Comment: corresponds to period of deforestation and strong erosion (Morand, 1971).

Gif-2779. Marais de Cessières, 108/65 $4220 \pm 140$ pected.

Peat $0.65 \mathrm{~m}$ below surface, Core 108. Comment: much older than ex-

Gif-3046. Marais de Cessières, 106/50

$2300 \pm 100$

Peat 0.45 to $0.5 \mathrm{~m}$ below surface, Core 106 . 
Gif-3047. Marais de Cessières, 104.50/50

$250 \pm 80$

Peat 0.45 to $0.5 \mathrm{~m}$ below surface, Profile 104. Comment: dates reappearance of Sylva, Pinus, and Ericaceum.

Gif-3048. Marais de Cessières, 104.51/50

$\mathbf{3 8 0} \pm \mathbf{8 0}$

Peat $0.5 \mathrm{~m}$ below surface, Profile 104 .

Gif-3049. Marais de Cessières, 104.44/50

$1080 \pm 90$

Peat $0.5 \mathrm{~m}$ below surface, Profile 104 .

Gif-2228. Marais de Cessières, 104.5/90

$2850 \pm 100$

Peat $0.9 \mathrm{~m}$ below surface, Profile 104 .

Gif-2230. Marais de Cessières, 104.2/305

$2820 \pm 100$

Peat $3.05 \mathrm{~m}$ below surface, Profile 104 .

Gif-3392. Marais de Cessières, 104.30/310

$4500 \pm 250$

Peat $3.1 \mathrm{~m}$ below surface, Profile 104 .

Gif-2780. Marais de Cessières, 102.1/690-700 $9100 \pm 300$

Peat 6.9 to $7 \mathrm{~m}$ below surface, Profile 102.1 .

Gif-2782. Marais de Cessières, $102.1 / 800-810 \quad 4890 \pm 140$

Peat 8 to $8.1 \mathrm{~m}$ below surface, Profile 102.1, Pre-boreal zone. Comment: age inversion between Gif-2782 and -2780 in Profile 102.1 is unexplained.

Gif-3390. Marais de Cessières, 102.11/810 $6720 \pm 130$

Peat $8.1 \mathrm{~m}$ below surface, Profile 102.11. Comment: either rate of peat formation is very variable in Cessières marsh or sample is contaminated.

Gif-3391. Marais de Cessières, 102.11/690 $\quad 8840 \pm 160$

Peat $6.9 \mathrm{~m}$ below surface, Profile 102.11. Comment: beginning of elm.

Gif.2783. Marais de Cessières, 706/750-760 $10,400 \pm 400$

Peat 7.5 to $7.6 \mathrm{~m}$ below surface, Core 706 . From bottom of 2 nd large acid peat bog of Cessières marsh.

Gif-3389. Marais de Cessières, $233 / 350 \quad 7870 \pm 140$

Peat $3.5 \mathrm{~m}$ below surface, Core 233 .

Gif-3393. Marais de Cessières, $743 / 285 \quad 9590 \pm 170$

Peat $2.85 \mathrm{~m}$ below surface, Core 743. Comment: Boreal-Pre-boreal transition.

General Comment: discrepancy sometimes observed between pollen analysis and ${ }^{14} \mathrm{C}$ chronology may be attributed to heterogeneity of peat bog and to water transport of pollen.

Forêt de Fontainbleau series, Seine et Marne

Vertical faces in large, currently worked sandpits, in forest of Fontainbleau show some superficial Quaternary formations (grèves) lying in situ on Stampian sand. These layered deposits of fine sand and gravels result 
from filling of valleys following formation of slopes from gelification (or frostwork) and solifluction (Puyfaucher and Collin, 1971). Bone fragments and well-defined brown organic horizons found in some secs were dated to establish chronology of alluvial periglacial Würmian formations in S Parisian basin. Area loc in Plaine de la Justice, $15 \mathrm{~km}$ SSW of La Ferté-Alais $\left(48^{\circ} 29^{\prime} \mathrm{N}, 20^{\circ} 21^{\prime} \mathrm{E}\right)$, Plaine de Chanfroy $\left(48^{\circ} 24^{\prime} \mathrm{N}, 2^{\circ} 34^{\prime} \mathrm{E}\right)$, and near Chailly-en-Bière $\left(48^{\circ} 27^{\prime} \mathrm{N}, 2^{\circ} 37^{\prime} \mathrm{E}\right)$ at $\mathrm{W}$ border of forêt de Fontainbleau.

\section{Gif-1905. Plaine de Chanfroy, C 1}

$22,100 \pm 700$

Fine humic sand at depth $4 \mathrm{~m}$. Coll and subm 1970 by F Morand. Comment: humic acids fraction dated, humin fraction absent. No traces of organic carbon, either soluble or insoluble, were found in overlying sand levels. This indicates that there is no infiltration of recent humic acids from superficial soil and, consequently, that dated organic carbon is probably not contaminated. Age of this level can be taken as true age.

\section{Gif-2509. Plaine de Chanfroy, 1}

$14,440 \pm 200$

Bison rib in fluvial sandy level at depth $2.2 \mathrm{~m}$ in same sand pit as Gif-1905. Coll and subm 1972 by A Puyfaucher, Inst Geog, Paris (Puyfaucher and Amat, 1972).

\section{Gif-2904. Plaine de la Justice, 16-8/1}

$30,200 \pm 1100$

Upper part of black clayey organic horizon, probably marsh deposit, at depth $3.5 \mathrm{~m}$. Coll and subm 1972 by A Puyfaucher. Comment: humin fraction dated.

Gif-2458. Plaine de la Justice, 16-8/11 $\geqslant 35,700$ dated.

Lower part of same horizon as Gif-2904. Comment: humin fraction

\section{Gif-2510. Chailly-en-Bière, 2/7-11}

$23,350 \pm 500$

Humic sandy level at depth $2.1 \mathrm{~m}$. Coll and subm 1972 by A Puyfaucher. Comment: humin fraction dated.

Gif-2913. Chailly-en-Bière, 2/7-11

22,860 上 500

Same level as Gif-2510. Comment: humic acids fraction dated. Agreement between dates for soluble and insoluble organic fractions of this paleosol confirms ages for fossil horizons in region.

Gif-2996. Chailly-en-Bière, 2

$$
20,900 \pm 1100
$$

Organic horizon, on millstone. Coll and subm 1974 by A Puyfaucher. Comment: humin fraction dated.

Gif-2459. Chailly-en-Bière, 2/5

Black organic horizon, overlain by fine sand and gravels, lying on white sand of Fontainbleau. Comment: humin fraction dated. 
General Comment: dates distinguish periods of periglacial climate from more temperate episodes, indicated by dated paleosols, during recent Würm.

Gif-2871. Erondelles, Somme

$$
2070 \pm 100
$$

Shells (Cardium edule) in sand underlain by peaty level, at depth $1 \mathrm{~m}$, S alluvial plains of low valley of Somme R $\left(50^{\circ} 5^{\prime} \mathrm{N}, 1^{\circ} 55^{\prime} \mathrm{E}\right)$. Coll and subm 1973 by M Ters, Inst Geog, Paris.

Gif-2872. Salenelles, Somme

Shells (Cardium edule) from sandy bar ca $4 \mathrm{~m}$ above msl $\left(50^{\circ} 12^{\prime} \mathrm{N}\right.$, $1^{\circ} 34^{\prime} \mathrm{E}$ ), Somme estuary. Coll and subm 1973 by M Ters.

Gif-1771. Camiers, Pas-de-Calais

$2910 \pm 100$

Sandy peat at top of beach between two brackish clay levels, $2.5 \mathrm{~m}$ above msl $\left(50^{\circ} 33^{\prime} \mathrm{N}, \mathrm{l}^{\circ} 35^{\prime} \mathrm{E}\right)$. Coll and subm by H Mariette, Samer, Pas-de-Calais.

\section{Eastern Channel series}

Peat from 3 cores from submarine peat bogs off Le Tréport $\left(49^{\circ} 59^{\prime}\right.$ $\mathrm{N}, \mathrm{I}^{\circ} 2 \mathrm{I}^{\prime} \mathrm{E}$ ), Eastern Channel. Coll and subm by J P Auffret, Dept Geol, Univ Caen.

Gif-2865. Core 890 (2)

$9870 \pm 200$

Peat, $21 \mathrm{~m}$ below msl.

Gif-2866. Core 890 (3)

$8800 \pm 170$

Peat, $20.8 \mathrm{~m}$ below msl.

Gif-2867. Core 585

$7470 \pm 150$

Peat, $18.7 \mathrm{~m}$ below msl.

General Comment: evidence of sea-level variations at beginning of postglacial. Dates show sea level was lower than $21 \mathrm{~m}$ before 8800 BP. Pollen analysis of cores increased understanding of vegetational history from late-glacial to Boreal-Atlantic transition (Huault and Auffret, 1975).

Gif-2860. Lingreville 1, Manche

$680 \pm 80$

Peaty level, $10 \mathrm{~cm}$ thick, in sand dune, $0.4 \mathrm{~m}$ above high sea level on shore $\left(48^{\circ} 57^{\prime} \mathrm{N}, 1^{\circ} 31^{\prime} \mathrm{W}\right)$. Coll and subm 1973 by J P Lautridou, Centre Geomorphol, CNRS, Caen. Comment: corresponds to "Dunkerqian III" transgression, in 13th century (Giresse and Lautridou, 1973).

Gif-2862. Lingreville 3, Manche

$3880 \pm 100$

Tree trunk in white sands, $1.5 \mathrm{~m}$ below high sea level, from beneath Lingreville 1. Coll and subm by $\mathrm{P}$ Lautridou. Comment: dates stage of Flandrian transgression.

\section{Dol-de-Bretagne series, Ille et Vilaine}

Flandrian deposits filling in depression corresponding to present marshes and polderlands around Dol-de-Bretagne $\left(48^{\circ} 33^{\prime} \mathrm{N}, 1^{\circ} 45^{\prime} \mathrm{W}\right)$, 
Ille et Vilaine, consist of marine sediments, sands, and silts with intercalated peat layers. ${ }^{14} \mathrm{C}$ and pollen analyses show that peaty layers were formed at end of Boreal period towards $8200 \mathrm{BP}$, at end of Atlantic, and beginning of Sub-boreal between 3900 and 3450 BP, and during Subatlantic, from 3000 в even regression) to explain peat growth but because of differential compaction of sediments under marshes, precise curve of variations of sea level cannot be drawn. Coll and subm 1970-1971 by G Delibrias. Pollen analysis by M T Morzadec, Univ Rennes (Delibrias and Morzadec, 1975). Measurements are above or below msl in meters.

Gif-957. Boring S 11, $+4.8 \mathrm{~m}$

$3100 \pm 110$

Freshwater peat. Pollen zone VIIb-VIII. Sub-boreal.

Gif-958. Boring S 11, +4m

$3100 \pm 110$

Freshwater peat. Pollen zone VIlb-VIII. Sub-boreal. Alnus abundant, Fagus present.

Gif-1835. Boring S 11, +1.8m

$4400 \pm 140$

End of salty peat level. Pollen zone VIIb. Sub-boreal.

Gif-953. Boring S 11, -1.1m

$4800 \pm 140$

Salty peat. Pollen zone VIIb. Sub-boreal.

Gif 954. Boring S 11, - 1.95m

$11,600 \pm 650$

Freshwater clayey peat. Pollen zone III. Postglacial, Pre-boreal.

Gif-1837. Boring S 12, -1.3m

$5500 \pm 150$

End of salty peat level. Pollen zone VIIb. Sub-boreal.

\section{Gif-955. Boring $\mathrm{S} 12,-1.7 \mathrm{~m}$}

$5600 \pm 150$

Salty peat. Pollen zone VIIa-VIIb. Sub-boreal.

Gif-1838. Boring S 12, -2.3m

$7050 \pm 170$

Salty peat. Pollen zone VIIa. Atlantic.

Gif-2185. Boring $S 7,+3.2 \mathrm{~m}$

$3000 \pm 100$

Brackish organic sand. Pollen zone late VIIb. Atlantic.

Gif-2186. Boring S 7, $+\mathbf{2 m}$

$3860 \pm 110$

Salty peat. Pollen zone VIIb.

Gif-2187. Boring $S$ 7, $-\mathbf{0 . 5 m}$

$5400 \pm 120$

Freshwater peat. Pollen zone VIIb. Atlantic.

Gif-2188. Boring $S 7,-10 \mathrm{~m}$

$8200 \pm 150$

Peaty clay. Pollen zone VIa. Boreal.

Gif-950. Boring S 10, $+2.15 \mathrm{~m}$

$3900 \pm 140$

Salty peat. Pollen zone VIIb. Sub-boreal. 
Gif-951. Boring $S$ 10, $-0.2 \mathrm{~m}$

$6750 \pm 170$

Salty peat. Pollen zone late VIIa. Atlantic.

Gif-952. Boring $S$ 10, -12m

$9800 \pm 230$

Peaty silt. Pollen zone VIc. Boreal.

Gif-2189. Boring S 8, $+1.8 \mathrm{~m}$

$3450 \pm 100$

Brackish organic sand. Pollen zone VIIb. Sub-boreal.

Gif-947. Boring S 8, +1m

$5450 \pm 150$

Salty peat. Pollen zone VIIb. Sub-boreal.

Gif-948. Boring S 8, $+0.25 \mathrm{~m}$

$5450 \pm 150$

Salty peat. Pollen zone late VIIb. Sub-boreal.

Gif-1833. Boring S 8, -9m

$10,100 \pm 230$

Organic clay. No pollen.

Gif-2184. Boring $S$ 1, $+\mathbf{1 . 8 m}$

$2390 \pm 100$

Peaty soil. Pollen zone late VIIb. Sub-boreal.

Gif-943. Boring S 1, +0.5m

$5200 \pm 150$

Salty peat. Pollen zone VIIa. Atlantic.

Gif-944. Boring S 1, -0.25m

$5000 \pm 150$

Salty peat. Pollen zone VIIa. Atlantic.

Gif-946. Boring S 1, -3m

$6800 \pm 170$

Marine shells in brackish sand.

Gif-931. Boring S 9, $+\mathbf{2 . 7} \mathrm{m}$

$360 \pm 90$

Organic soil. Pollen zone VIII. Sub-atlantic.

Gif-935. Boring S 9, 0.4m

$4360 \pm 140$

Salty peat. Pollen zone VIIa-VIIIb. Limit Sub-boreal-Atlantic.

Gif-936. Boring S 9, +0.2m

$4480 \pm 140$

Salty peat. Pollen zone VIIa. Atlantic.

Gif-1834. Boring S 9, $-\mathbf{0 . 1 m}$

$5850 \pm 150$

Salty peat. Pollen zone VIIa. Atlantic.

Gif-937. Boring S 9, $-\mathbf{0 . 2 m}$

$4250 \pm 140$

Salty peat. Pollen zone VIIa. Atlantic.

Gif-940. Boring S 9, -9.3m

$6900 \pm 150$

Broken shells in marine sand. No pollen.

Gif-941. Boring S 9, $-10.2 \mathrm{~m}$

$6200 \pm 150$

Salty peat. Pollen zone VIc. Boreal.

$11,600 \pm 270$

Gif-942. Boring S 9, -15m

Broken shells in fluviatile sand. 
Gif-1879. Boring S 2, $+3.5 \mathrm{~m}$

$4400 \pm 140$

Salty peat. Pollen zone VIIa. Atlantic.

\section{Ver-sur-Mer series, Calvados}

Peat from littoral peat bog cropping out on beach $\left(49^{\circ} 20^{\prime} \mathrm{N}, 0^{\circ} 30^{\circ}\right.$

W). Coll and subm 1972 by C Larsonneur, Inst Geol, Univ Caen.

Gif-2693. $\quad$ V 12

$1080 \pm 100$

Pollen zone, Sub-atlantic.

Gif-2695. V 32

$1580 \pm 100$

Pollen zone, Sub-atlantic.

Gif-2694. V 28

$2850 \pm 110$

Pollen zone, end of Sub-boreal.

General Comment: outcrop overlies archaeol level with Neolithic industry.

\section{Saint Guinoux series, Dol, Ille et Vilaine}

Peat from tidal marsh of Dol at Saint Guinoux $\left(48^{\circ} 33^{\prime} \mathrm{N}, 1^{\circ} 45^{\prime} \mathrm{W}\right)$. Coll and subm 1970 by F Verger, Ecole Pratique Haute Etudes, Paris (Verger, 1971).

Gif-1686. Saint Guinoux, Dol 81

$2720 \pm 110$

Peat at depth 1.7 to $1.95 \mathrm{~m}, 1.1 \mathrm{~km}$ SSW of Saint Guinoux under ancient channel with reversed relief.

Gif-1687. Saint Guinoux, Dol A

Peat at depth 2.8 to $2.9 \mathrm{~m}, 1 \mathrm{~km}$ SW of Saint Guinoux, under ancient channel with unreversed relief. Coll and subm 1970 by F Verger.

General Comment: dated as part of geomorphol study of region; indicates relief inversion between 3500 and $2700 \mathrm{BP}$.

\section{Saint Marc-en-Treveneuc series, Côtes du Nord}

Peat and wood samples from cores in large peat bog covering foreshore in small valley mouth, in cove of Saint Marc-en-Treveneuc $\left(48^{\circ} 40^{\prime}\right.$ $\left.\mathrm{N}, 2^{\circ} 50^{\prime} \mathrm{W}\right)$. Coll and subm 1971-1978 by M Ters. Deposit is ca $15 \mathrm{~m}$ thick accumulation of peaty and sandy sediments. Dates enable study of sea level rises and development of vegetation during Holocene. Alts are related to msl. Present tidal range on coast is $12.85 \mathrm{~m}$.

Gif-2979. S 2-14

Peat, $+7.85 \mathrm{~m}$.

Gif-2607. M XII

Peat, $+6.35 \mathrm{~m}$.

Gif-4523. D B

$3820 \pm 110$

Peat, $+4.75 \mathrm{~m}$. 
Gif-4417. Fu 37

$4500 \pm 110$

Peat, $+4.2 \mathrm{~m}$.

Gif-2606. M XII

$5080 \pm 140$

Peat, $+1.15 \mathrm{~m}$.

Gif-4416. A 8

$5600 \pm 120$

Tree trunk, $+0.5 \mathrm{~m}$.

Gif-4524. D B

$5790 \pm 130$

Peat, $+0.35 \mathrm{~m}$.

Gif-2978. B 2-2

$6070 \pm 130$

Peat, $-1.9 \mathrm{~m}$.

Gif-2152. I

$6400 \pm 130$

Tree trunk, $-1.85 \mathrm{~m}$.

Gif-2527. 43

$7050 \pm 160$

Peat, $-5.95 \mathrm{~m}$.

Gif-3792. R 20

$7060 \pm 160$

Peat, $-7.3 \mathrm{~m}$.

Gif-3097. C M 38

$7370 \pm 140$

Peat, $-7 \mathrm{~m}$.

General Comment: palynol study indicates: upper part of peat, +7.45 to $\mathrm{rm}$ is Sub-atlantic and Sub-boreal. Lower part of peat and underlying clay are Atlantic. Some short-term oscillations of sea level during Holocene transgression were established, high levels ca $6200 \mathrm{BP}$ at $-8 \mathrm{~m}$, са $4900 \mathrm{BP}$ at $-5 \mathrm{~m}$, са $3350 \mathrm{BP}$ at $-2.5 \mathrm{~m}$, and low level occurred ca $7400 \mathrm{BP}$ at $-15 \mathrm{~m}$, just before minor transgression (Ters, 1973).

\section{Anse du Palus series, Côte du Nord}

Peat from peaty layer lying on shelly sand, $2 \mathrm{~m}$ below msl $\left(48^{\circ} 40^{\prime} \mathrm{N}\right.$, $\left.2^{\circ} 56^{\prime} \mathrm{W}\right)$. Coll and subm 1972 by $M$ Ters.

Gif-3474. Job 1

$3580 \pm 110$

Peaty black clay from top of peaty layer.

Gif-3475. Job 44

$5330 \pm 140$

Peat from base of peaty layer.

Gif-2953. Croesty-en-Arzon, Morbihan

$3130 \pm 100$

Peaty clay under marine sand, in marsh $\left(47^{\circ} 32^{\prime} \mathrm{N}, 2^{\circ} 54^{\prime} \mathrm{W}\right)$. Coll and subm 1973 by M Ters. Comment: dates continental episode correlated with minor regression of sea.

\section{Gif-2528. Saint-Nazaire, Loire Atlantique}

Oyster shells at depth $30 \mathrm{~m}$ in sediments from Loire valley $\left(47^{\circ} 17^{\prime} \mathrm{N}\right.$, $\left.2^{\circ} 10^{\prime} \mathrm{W}\right)$. Coll and subm 1972 by $\mathrm{M}$ Ters. Comment: estimates rate of valley filling. 


\section{Brétignolles series, Vendée}

Wood and peat on foreshore of beach of Brétignolles $\left(46^{\circ} 38^{\prime} \mathrm{N}, 1^{\circ}\right.$ $52^{\prime}$ W). Coll and subm 1972-1974 by M Ters.

Gif-3421. 85R

$3430 \pm 110$

Tree trunk in situ, $1 \mathrm{~m}$ above msl, lying on peat dated at $4990 \pm 120$ (Gif-2109) and $4900 \pm 1120$ (Gif-2108) (R, 1974, v 16, p 65).

Gif-2525. $\quad 14$

$5900 \pm 140$

Peat, $4 \mathrm{~m}$ below msl.

Gif-3420. La Grande Poissolière, Vendée

$7600 \pm 170$

Wood in peat layer intercalated between periglacial alluvium of Auzance $\mathrm{R}$ and Holocene alluvium inland $\left(46^{\circ} 24^{\prime} \mathrm{N}, 1^{\circ} 40^{\prime} \mathrm{W}\right)$. Coll and subm 1974 by M Ters. Comment: dates beginning of valley filling resulting from Holocene rise of sea.

\section{Sonneville series, Charente}

Typical formations of Charente region composed of deposits of alternatingly fine- and coarse-layered sands are called "grèzes litées". They result from action of snow on slopes in limestone environment and from drift caused by a sheet flood phenomenon during periglacial period. These sandstone-like formations are often cut by organic horizons, pseudo-paleosols, resulting from soils developed on high part of slope during favorable climatic periods and swept down slope by action of snow when climatic conditions deteriorated. Dates obtained from mollusk shells and organic matter from paleosols. Coll in sandpit of Sonneville $\left(45^{\circ} 35^{\prime} \mathrm{N}, 0^{\circ} 11^{\prime} \mathrm{W}\right)$ and subm 1972-1975 by Y Guillien, CNRS, Paris (Guillien and Puissegur, 1969; Guillien, 1975).

\section{Gif-2417. Sonneville 3}

Mollusk shell from basal level of Upper Mesowürmian Paleosol 3. Comment: molluscan fauna is characteristic of cold steppe.

\section{Gif-2675. Sonneville 3}

Humin fraction from top of Upper Paleosol 3, same level as mollusks (Gif-2417). Comment: soluble organic fraction dated at 27,080 \pm 350 , Gif-2675bis, $\delta^{13} \mathrm{C}=-25.3 \%$. Good agreement between two dates indicates that organic horizon is free of recent contamination.

Gif-2951. Sonneville 3

Humin fraction coll from same level as Gif-2675.

Gif-3423. Sonneville 3

Humin fraction from basal level of Upper Paleosol 3. Comment: agrees within limits of statistical errors with other dates from this soil. Humic acids absent.

Gif-3274. Sonneville 2

Humin fraction from paleosol above Sonneville 3. Comment: humic acids absent. 
Gif-3273. Sonneville $1 a+1 b$

$21,600 \pm 600$

Humin fraction from paleosol above Sonneville 2. Comment: humic acids absent.

Gif-3537. Sonneville II-III

$8790 \pm 400$

Humin fraction from more superficial organic horizon. Comment: very low percentage of organic $\mathrm{C}, 0.06 \%$.

Gif-3538. Sonneville II-III, 20-21-22 $9230 \pm \mathbf{4 0 0}$

Humin fraction, from same level as Gif-3537. Comment: very low percentage of organic C, $0.1 \%$. Date confirms that of Gif-3537. Obvious circulation of water through this level suggests recent contamination.

General Comment: dates approx end of soil development period and change in climatic conditions that initiated slope instability. Sonneville dates generally agree with known chronology for Upper Pleniglacial in Europe. Well-dated $1.4 \mathrm{~m}$ thick Upper Paleosol 3, corresponds to important Würmian Interstadial ca 26,000 BP.

\section{Charente valley series, Charente Maritime}

Samples from muddy sediments of lower valley of Charente $\mathrm{R}$, Charente Maritime. Coll and subm 1975 by C Gabet, Rochefort, Charente Maritime.

Gif-3757. Brouage 1 $\left.44^{\prime} \mathrm{W}\right)$.

Shells from shelly sand, $20 \mathrm{~m}$ below msl at Brouage $\left(45^{\circ} 52^{\prime} \mathrm{N}, 0^{\circ}\right.$

Gif-3758. Brouage 2

$1790 \pm 100$

Shells from shelly sand, $23 \mathrm{~m}$ below msl.

Gif-3756. Rochefort, Vieille Forme

$3530 \pm 110$

Shells from shelly sand, $25.4 \mathrm{~m}$ below msl $\left(45^{\circ} 57^{\prime} \mathrm{N}, 0^{\circ} 57^{\prime} \mathrm{W}\right)$.

Gif-3755. Rochefort, Corderie $\left.57^{\prime} \mathrm{W}\right)$.

Peaty sand overlying black clay, $28.2 \mathrm{~m}$ below msl $\left(45^{\circ} 56^{\prime} \mathrm{N}, 0^{\circ}\right.$

General Comment: samples dated to trace Holocene transgression on coast, but precise sea level is difficult to deduce from depth of these samples coll in marshes and tidal channels where near-shore sedimentation is important factor. Dates show formation of estuary of Charente R.

\section{Gif-2555. Marsh of Arvert, Saintonge, Charente \\ Maritime}

$4290 \pm 120$

Shells from ancient littoral offshore bar, $9 \mathrm{~m}$ below msl $\left(45^{\circ} 47^{\prime} \mathrm{N}\right.$, $\left.1^{\circ} 15^{\prime} \mathrm{W}\right)$. Coll and subm 1972 by C Gabet (1978). Comment: dates stage in formation of Marsh of Saintonge; difficult to relate to precise sea level.

\section{La Pérroche series, Oléron Island}

Salty peat bog on strand overlying marine sand layer, $\mathrm{S}$ La Pérroche $\left(46^{\circ} 54^{\prime} \mathrm{N}, 4^{\circ} 18^{\prime} \mathrm{W}\right)$, Charente Maritime. Coll and subm 1972 by $\mathrm{C}$ Gabet (1974). 
Gif-2679. La Pérroche 1

Peat at base, $1.3 \mathrm{~m}$ below msl.

Gif-2680. La Pérroche 2

Peat at $0.3 \mathrm{~m}$ below msl.

Gif-4234. La Pérroche 3

Peat from upper layer.

General Comment: dates agree with known sea-level variations during Holocene on British and Channel littorals.

Gif-2987. Tonnay, Charente

$3660 \pm 110$

Oyster shells from shelly deposit $0.6 \mathrm{~m}$ thick, at $9 \mathrm{~m}$ depth in maritime plain of Charente $\left(45^{\circ} 57^{\prime} \mathrm{N}, 0^{\circ} 53^{\prime} \mathrm{W}\right)$. Coll and subm by C Gabet. Comment: dates stage in formation of maritime plain of Charente. Dates a little younger than expected from Neolithic industry assoc; difficult to relate to precise sea level.

\section{Saint Georges d'Oléron series, Oléron Island}

Littoral deposits from Marais des Salines $\left(45^{\circ} 58^{\prime} \mathrm{N}, 2^{\circ} 16^{\prime} \mathrm{W}\right)$. Coll and subm 1974 by $\mathrm{C}$ Gabet to determine stages of forward advance of coast in NE part of isle.

Gif-3382. Marais des Salines, 1

Marine shells from offshore bar, La Moulinade $2 \mathrm{~km}$ inland from coast, $2 \mathrm{~m}$ above msl.

Gif-3383. Marais des Salines, 2

Peat from sandy peat in marsh between shelly deposits, $4 \mathrm{~m}$ below msl, on offshore bar.

Gif-4393. Marais des Salines, 3

$2710 \pm 100$

Marine shells from offshore bar off La Moulinade, $1.5 \mathrm{~m}$ above msl.

Gif-4394. Marais des Salines, 4

Modern

Marine shells in marsh at foot of offshore bar, La Moulinade. Comment: shells probably deposited by very high tide.

Gif-4395. Marais des Salines, 5

$$
700 \pm 90
$$

Marine shells from offshore bar des Arceaux, 2.5m above msl.

General Comment: dates trace formation of Marais des Salines, built from deposition of three shelly bars which were remains of ancient shorelines and by sediment-filled pond that formed between bars. Dates indicate stages of coastal aggradation in NE part of Oléron I.

Gif-4418. Oléron Island

$3430 \pm 110$

Peat layer between two marine deposits $0.5 \mathrm{~m}$ below msl, in filling of small valley, Oléron I. Coll and subm by M Ters. Comment: dates minor regression of sea between two transgressive episodes. Probably dates same fluctuations of sea as Gif-2953 and -3421. 
Gif-2767. Bidart, Pyrénées Atlantiques

Wood from fossil tree trunk in coastal alluvial nappe deformed by tectonics, at Bidart $\left(43^{\circ} 26^{\prime} \mathrm{N}, 1^{\circ} 36^{\prime} \mathrm{W}\right)$. Coll and subm 1973 by $\mathrm{G}$ Viers, Fac Lettres, Toulouse. Comment: date confirms old age of this formation.

\section{B. Spain and Portugal}

Gif-2707. La Franca, Asturias

Peat overlying marine pebble deposit on marine abrasion surface, covered by consolidated marine material 6 to $8 \mathrm{~m}$ above msl, on beach of La Franca $\left(43^{\circ} 23^{\prime} \mathrm{N}, 4^{\circ} 36^{\prime} \mathrm{W}\right)$. Coll 1970 and subm 1973 by G Mary, Coll Sci Univ, Le Mans. Comment: confirmed expectation that this is old level (probably Tyrrhenniam III) and not result of recent tectonic movements. Pollen analysis: Betula and Alnus dominant.

\section{Llano Ronanzas series, Asturias}

Peaty formation, $2.5 \mathrm{~m}$ thick, on remote plateau at alt $250 \mathrm{~m}$ in hinterland, Llano Ronanza $\left(43^{\circ} 23^{\prime} \mathrm{N}, 4^{\circ} 23^{\prime} \mathrm{W}\right)$, La Borbolla. Coll 1970 and subm 1972 by $\mathrm{G}$ Mary.

Gif-2337. Llano Ronanzas $350 \pm 80$

Peat at 0 to $0.2 \mathrm{~m}$ above surface.

\section{Gif-2336. Llano Ronanzas}

$1450 \pm 90$

Peat at 1.1 to $1.5 \mathrm{~m}$ above surface.

\section{Gif-2335. Llano Ronanzas}

$3210 \pm 100$

Peat at 2.2 to $2.5 \mathrm{~m}$ above surface.

General Comment: Corylus abundant at all levels.

\section{Asturian coastal series, Spain}

Peat on beach and cliff of Jerra $\left(43^{\circ} 28^{\prime} \mathrm{N}, 3^{\circ} 48^{\prime} \mathrm{W}\right)$ and of San Vicente de la Barquera $\left(43^{\circ} 23^{\prime} \mathrm{N}, 4^{\circ} 24^{\prime} \mathrm{W}\right.$ ) on Asturian coast bordering Bay of Biscay, N Spain. Coll and subm 1970 to 1972 by G Mary.

Gif-2635. Jerra 1

$5880 \pm 130$

Peat from outcropping $60 \mathrm{~cm}$ above present bar at cliff base. Comment: indicates marine environment.

Gif-1893. Jerra 2

Submerged peat with hazelnuts, $25 \mathrm{~m}$ from foot of cliff.

Gif-2917. Jerra 3

$5300 \pm 120$

Peat close to Gif-1893.

Gif-3050. Jerra 4.

$5850 \pm 200$

Submerged peat, $150 \mathrm{~m}$ from foot of cliff.

\section{Gif-2636. Rio Bederna, San Vicente de la}

Barquera Beach

Peat bog on pebble bar, 1.1 to $2 \mathrm{~m}$ above high sea level, exposed at mouth of Rio Bederna. 
General Comment: Asturian coast, contrary to French coast, has kept traces of recent high sea levels (Mary, Médus, and Delibrias, 1975). Pollen in dated peats would indicate phytodynamic delay during Holocene with regard to French Basque country.

\section{Torremolinos series, Spain}

Shells from marine formation at Torremolinos $\left(36^{\circ} 38^{\prime} \mathrm{N}, 4^{\circ} 30^{\prime} \mathrm{W}\right)$, near Malaga. Coll and subm 1972 by R Lhenaff, Inst Geog, Lille.

Gif-2690. Torremolinos 1

$1350 \pm 90$

Shells from bottom of red detrital continental formation, lying on lumachelle.

Gif-2691. Torremolinos 2

$\geqslant \mathbf{3 0 , 0 0 0}$

Shells in lumachelle, 1 to $2 \mathrm{~m}$ above msl.

\section{Azores series, Portugal}

Core K 11, 3550m depth (36 47' $7^{\prime \prime} \mathrm{N}, 27^{\circ} 22^{\prime} 3^{\prime \prime} \mathrm{W}$ ), near Azores Islands, Atlantic Ocean. Subm 1972 by L Pastouret, CNEXO, Plouzané. Total inorganic carbon was used to date samples.

Gif-2522. 5 to $16 \mathrm{~cm}$

$$
\begin{aligned}
4150 & \pm 110 \\
21,850 & \pm 570
\end{aligned}
$$

Gif-2523. 61 to $75 \mathrm{~cm}$

\section{Linz series}

\section{Austria}

Fossil wood from recent alluvial plains of Donau R, in Linz region. Coll by H Kohl and subm 1971 by M Leger, Inst Geog, Paris (Leger, 1965).

Gif-2252. Pleschinger Au, 63

$1490 \pm 90$

Fossil wood (Fagus sp), at depth 6 to $7 \mathrm{~m}$ in alluvial plain of Donau $\left(48^{\circ} 19^{\prime} \mathrm{N}, 14^{\circ} 20^{\prime} \mathrm{E}\right)$.

Gif-2253. Pleschinger Au, 69

$1440 \pm 90$

Fossil wood (Ulmus sp), at depth 6 to $7 \mathrm{~m}$ in alluvial plain of Donau.

Gif-2251. Pleschinger Au, 52

$3640 \pm 110$

Fossil wood (Quercus sp), at depth 6 to $7 \mathrm{~m}$ in alluvial plain of Donau.

Gif-2249. Asten Fisching, 9

$2390 \pm 100$

Fossil wood (Fraximus sp), at depth 10 to $11 \mathrm{~m}$ in lowest terrace at Asten Fisching $\left(48^{\circ} 14^{\prime} \mathrm{N}, 14^{\circ} 25^{\prime} \mathrm{E}\right)$.

Gif-2250. Asten Fisching, 10

$2600 \pm 100$

Fossil wood (Ulmus sp), at depth 10 to $11 \mathrm{~m}$ in lowest terrace.

Gif-2254. Hirschgasse, Linz, 75

$12,100 \pm 220$

Fossil wood (Populus sp), in organic horizon in silty upper deposit of low terrace, at $2 \mathrm{~m}$ depth, Hirschgasse $\left(48^{\circ} 18^{\prime} \mathrm{N}, 14^{\circ} 16^{\prime} \mathrm{E}\right)$. 


\section{North Africa}

Gif-1891. Sebkhet-en-Noual, Tunisia

$300 \pm 90$

Ostrich egg shell from ground surface at top of terrace, Sebkhet-enNoual $\left(34^{\circ} 24^{\prime} \mathrm{N}, 9^{\circ} 52^{\prime} \mathrm{E}\right)$. Coll and subm 1970 by J P Ballais, Univ Caen. Comment: indicates recent existence of ostrich in region.

\section{Bou-Regreg series, Morocco}

Oyster shells in marine alluvium in mouth of Bou-Regreg wadi near Rabat $\left(34^{\circ} 02^{\prime} \mathrm{N}, 6^{\circ} 51^{\prime} \mathrm{W}\right)$. Coll and subm 1973 by J Riser, Fac Sci St Jerôme, Marseille.

\section{Gif-2851. Bou-Regreg 1}

$\mathbf{8 7 7 0} \pm \mathbf{2 0 0}$

From base of oyster shell level, 25 to $1 \mathrm{~m}$ below msl.

Gif-2852. Bou-Regreg 3

$6490 \pm 160$

From top of same oyster shell level.

General Comment: dates corroborate rapid rise of sea level during "Mellahien" (Flandrian on these coasts).

\section{E. West Africa}

Dakar, Core $40 \mathrm{~S} 43$ series

Samples from marine Core $40 \mathrm{~S} 43,4.2 \mathrm{~km}$ off Dakar $\left(14^{\circ} 36^{\prime} 60^{\prime \prime} \mathrm{N}\right.$, $17^{\circ} 14^{\prime} 58^{\prime \prime} \mathrm{W}$ ), at depth $15.8 \mathrm{~m}$, in old marigot (estuarine marsh). Coll by Mission Rosilda and subm 1973 to date Holocene transgression.

Gif-2824. 340 to $330 \mathrm{~cm}$

$$
8400 \pm 180
$$

Clayey peat with coarse sand from base of peat at bottom of core.

Gif-2856. 300 to $290 \mathrm{~cm}$

$8180 \pm 180$

Oyster shells from upper part of peat level.

Gif-2823. 40 to $30 \mathrm{~cm}$

$8150 \pm 180$

Peaty clay from top of core, separated from low peaty horizon by $1.5 \mathrm{~m}$ oyster mud level.

General Comment: dates suggest very rapid rise of sea level са 8000 вр.

\section{Louti series, Cameroon}

Calcareous nodules in vertisol from low terrace of right bank of Mayo Louti R $\left(10^{\circ} 46^{\prime} \mathrm{N}, 13^{\circ} 55^{\prime} \mathrm{E}\right)$, at Figuil, Benoue. Coll and subm 1971 by A Marliac, ORSTOM, Yaounde, Cameroon.

\section{Gif-2233. Louti, 70 AK 911}

$$
\mathbf{8 4 0 0} \pm 110
$$
at depth $2 \mathrm{~m}$, Profile 9 . Site subject to seasonal floods.

\section{Gif-2234. Louti, 70 AK 9111}

$14,720 \pm 200$

Calcareous nodules $50 \mathrm{~cm}$ below Gif-2233, Profile AK 9. Site subject to seasonal floods. 
Gif-2235. Louti, 71 F 2 bis 111 a

$9480 \pm 120$

Calcareous nodules in gray-black vertisol, overlain by colluvium at depth $4 \mathrm{~m}$, Profile F 2. Above seasonal flood level.

Gif-2236. Louti, 71 F 2 bis 111 b

$15,320 \pm 210$

Calcareous nodules from $90 \mathrm{~cm}$ below Gif-2235, Profile F 2 .

Gif-2237. Louti, 71 F 2 bis V c

$8690 \pm 110$

Calcareous nodules from yellow sandy-silty horizon at depth $3 \mathrm{~m}$ under Gif-2236, Profile F 2. Site may be subject to floods.

\section{Gif-2238. Louti, 71 F 4111 a}

$7030 \pm 100$

Calcareous nodules in gray-black vertisol in sandy gritty colluvium at depth $7 \mathrm{~m}$, Profile F 4. Above seasonal flood level.

Gif-2239. Louti, 71 F 4 V b

$6450 \pm 90$

Calcareous nodules from $5 \mathrm{~m}$ below Gif-2238, Profile 4. Above seasonal flood level.

Gif-2240. Louti, 71 F 4 V

Modern

Organic matter from vertisol just above nodules Gif-2239, Profile 4. Comment: no carbonate in this level. Organic carbon content $0.1 \%$; inactive $\mathrm{CO}_{2}$ added for measurement.

General Comment: dates are inconsistent both within same profile and between profiles which may be caused by presence of Cretaceous calcareous sediment in part of drainage basin, and by active formation of calcareous deposits in profiles (F4 and F2 bis) observed since 1971. Modern age of organic matter from base of Profile F4, though not explained (Gif-2240) shows contamination of these levels. Study indicates unreliability of ${ }^{14} \mathrm{C}$ ages for this type of material in equatorial regions.

York Island series, Sierra Leone

Vermets from York I. $\left(8^{\circ} 30^{\prime} \mathrm{N}, 13^{\circ} 17^{\prime} \mathrm{W}\right)$. Coll and subm 1971 by J Laborel, Fac d'Aix, Marseille. Alts are above biol level of living vermets.

Gif-2404. York I., SL 1

$650 \pm 90$

Fossil formation, $1 \pm 0.5 \mathrm{~m}$, found in fissure.

Gif-2405. York I., SL 2

$1420 \pm 100$

Fossil formation, $2 \pm 0.5 \mathrm{~m}$.

Gif-2406. York I., SL 3

$740 \pm 90$ York I.

Fossil formation, $1.5 \pm 0.5 \mathrm{~m}$ from Sussex Islet, between Freetow and

General Comment: if compared with results obtained for fossil vermets along coast of Brazil at same date, sea level seems 0.5 to $1 \mathrm{~m}$ higher on W African coast (Laborel and Delibrias, 1976). 


\section{Ivory Coast series}

Shells coll and subm 1973 by R Pomel, Dept Géog, Univ ClermontFerrand, to date recent minor transgression of littoral zone of Ivory Coast.

Gif-2864. Dabou 1

$$
2020 \pm 100
$$

Shells from shelly sand mounds (faluns) with lagoon shells and anthropol deposits, at depth $2.5 \mathrm{~m}$, Dabou $\left(5^{\circ} 19^{\prime} \mathrm{N}, 4^{\circ} 23^{\prime} \mathrm{W}\right)$.

Gif-2863. Dabou 11

$1970 \pm 100$

Shells from basal level of same shelly mounds as Gif-2864.

Gif-3377. N'Gaty

$1950 \pm 90$

Shells from falun, N'Gaty $\left(5^{\circ} 19^{\prime} \mathrm{N}, 4^{\circ} 21^{\prime} \mathrm{W}\right)$.

Gif-3379. Bandama

Oyster shell from falun on right bank of Bandama $\mathrm{R}$ near Lahou $\left(5^{\circ} 08^{\prime} \mathrm{N}, 4^{\circ} 49^{\prime} \mathrm{W}\right)$.

Gif-3360. Dighoue

Oyster shell in falun, Digboue lagoon, near Balmer $\left(4^{\circ} 43^{\prime} \mathrm{N}, 6^{\circ} 39^{\prime}\right.$ W). Comment for Gif-2864, -2863, -3360, -3377, -3379: samples date formation of these anthropol deposits, positions of which suggest minor transgression.

Gif-3361. Grand-Berebi $\left.55^{\prime} \mathrm{W}\right)$.

Vermetidae, $0.5 \mathrm{~m}$ above high sea level, Grand-Berebi $\left(4^{\circ} 39^{\prime} \mathrm{N}, 6^{\circ}\right.$

Gif-3362. Dogbale 1

Vermetidae, $1.5 \mathrm{~m}$ above high sea level, Dogbale-Oulidie $\left(4^{\circ} 37^{\prime} \mathrm{N}\right.$, $\left.6^{\circ} 57^{\prime} \mathrm{W}\right)$.

Gif-3363. Doghale 2

$1920 \pm 90$

Vermetidae, $3 \mathrm{~m}$ above high sea level.

\section{South Benin series}

Samples to study recent Holocene formation of $\mathrm{S}$ Benin, shoreline resulting from fluvial, lagoonal, and estuarine sedimentation.

Gif-2847. N Ahémé Lake

$5700 \pm 140$

Peat at depth 160 to $200 \mathrm{~cm}$ in Core $\mathrm{L} 4 \mathrm{~b}-1$, ca $1 \mathrm{~m}$ above msl, from peat bog in ancient mangrove lagoon, probably developed at time of max of Nouakchottian transgression, $\mathrm{N}$ Ahémé Lake $\left(6^{\circ} 36^{\prime} \mathrm{N}, 2^{\circ} 00^{\prime} \mathrm{E}\right)$. Coll and subm 1973 by P Germain, Lab Radioecol, Cherbourg (Germain, 1975).

Gif-2848. S Porto-Novo

$5430 \pm 140$

Peat at depth 150 to $200 \mathrm{~cm}$ in Core P N 2 C-7, at ca msl, S PortoNovo $\left(6^{\circ} 26^{\prime} \mathrm{N}, 2^{\circ} 36^{\prime} \mathrm{E}\right)$. Coll and subm 1973 by $\mathrm{P}$ Germain. 
Gif-2849. Near Cotonou, C 1

Shells at depth 630 to $660 \mathrm{~cm}$ in Core C 1,1 to $2 \mathrm{~m}$ below msl, in sandy seastrand near Cotonou $\left(6^{\circ} 21^{\prime} \mathrm{N}, 2^{\circ} 25^{\prime} \mathrm{E}\right)$. Coll and subm 1973 by $\mathbf{P}$ Germain.

Gif-2850. Near Cotonou, $\mathbf{S} 3$

$2750 \pm 100$

Shells at depth 970 to $1070 \mathrm{~m}$ in Core S 3, ca $6 \mathrm{~m}$ below msl in S sandy seastrand near Cotonou $\left(6^{\circ} 21^{\prime} \mathrm{N}, 2^{\circ} 26^{\prime} \mathrm{E}\right)$. Coll and subm by $\mathrm{P}$ Germain. Comment: Gif-2849 and -2850 date levels corresponding to transition between fine marine sandy deposit and coarser sedimentation which marks beginning of formation of littoral seastrand.

Gif-3347. Bembe, P N 4 a

$2670 \pm 100$

Shells in black clay from fluvial-lagoonal formation on left bank of Oueme R at Bembe $\left(6^{\circ} 32^{\prime} \mathrm{N}, 2^{\circ} 32^{\prime} \mathrm{E}\right)$. Coll and subm 1974 by G Paradis, Univ Benin, Cotonou.

Gif-3348. Zoumbodji

$1250 \pm 90$

Shells (Arca senelis), N coastal lagoon, Zoumbodji $\left(6^{\circ} 20^{\prime} \mathrm{N}, 2^{\circ} 06^{\prime}\right.$ E). Coll and subm 1974 by $G$ Paradis.

Gif-3972. Cotonou, 13

$3200 \pm 100$

Marine shells in coarse littoral sand, 12 to $13.5 \mathrm{~m}$ depth, ca $6 \mathrm{~m}$ below msl, Cotonou $\left(6^{\circ} 21^{\prime} \mathrm{N}, 2^{\circ} 26^{\prime} \mathrm{E}\right)$. Coll and subm 1976 by $\mathrm{G}$ Paradis.

Gif-3974. Cotonou, 15

$1490 \pm 90$

Marine shells from shelly beds, $1.5 \mathrm{~m}$ thick, in gray sand $1 \mathrm{~m}$ above msl, from ancient beach $\left(6^{\circ} 21^{\prime} \mathrm{N}, 2^{\circ} 23^{\prime} \mathrm{E}\right)$. Coll and subm by G Paradis.

Gif-3973. Kraké

$830 \pm 90$

Charcoal with pottery assoc at depth $0.6 \mathrm{~m}$, in sandy seastrand, Kraké $\left(6^{\circ} 23^{\prime} \mathrm{N}, 2^{\circ} 41^{\prime} \mathrm{E}\right)$. Coll and subm 1976 by G Paradis. Comment: sample of archaeol interest.

Gif-3349. Kpodji 1a $970 \pm 90$

Shells (Mactra sp), from shelly sand level beneath clay, Kpodji $\left(6^{\circ} 23^{\prime}\right.$ N, $\left.2^{\circ} 35^{\prime} \mathrm{E}\right)$, Dahomey. Coll and subm 1974 by G Paradis.

Gif-3350. Kpodji 1 b $1120 \pm 90$

Shells (Pachymeliana aurita), from same level as Gif-3349.

General Comment: samples date environmental changes shown by sediment and faunal studies (Paradis, 1975; 1978) resulting from change in lagoon formation.

Ponta de San Jeronimo series, Sao Thome Island, Gulf of Guinea

Lithotamnies from raised beach at Ponta San Jeronimo $\left(0^{\circ} 19^{\prime} 55^{\prime \prime} \mathrm{N}\right.$, $\left.6^{\circ} 44^{\prime} 50^{\prime \prime} \mathrm{E}\right)$. Coll by $\mathrm{M}$ Cornen and subm 1973 by $\mathrm{P}$ Giresse, Univ Perpignan.

Gif-2998. Ponta de San Jeronimo, S T 1 $3900 \pm 100$

Shells from base of raised beach, $0.5 \mathrm{~m}$ above msl. 
Gif-2999. Ponta de San Jeronimo, S T 2

$2300 \pm 100$

Shells from top of raised beach, $1.5 \mathrm{~m}$ above msl.

General Comment: island is tectonically active and not suitable for study of small variations in sea level.

Gif-2948. Pointe Noire, Congo

$$
1890 \pm 90
$$

Charcoal lying on ocher sands covered by white sands at 5 to $7 \mathrm{~m}$ above msl ( $\left.4^{\circ} 46^{\prime} 30^{\prime \prime} \mathrm{S}, 11^{\circ} 52^{\prime} 10^{\prime \prime} \mathrm{E}\right)$. Coll and subm 1973 by P Giresse. Comment: dates deposition of white sands at end of Holocene.

\section{Kouilou estuary series, Congo}

Six $30 \mathrm{~m}$ borehole cores were taken in Holocene filling of Kouilou estuary valley to study most recent shoreline oscillations. Coll and subm 1974 by P Giresse.

\section{Gif-3451. Kouilou estuary, BK $1 \mathrm{D} \quad 610 \pm 90$}

Organic remains in black clay, $6 \mathrm{~m}$ below high sea level $\left(4^{\circ} 28^{\prime} 40^{\prime \prime} \mathrm{S}\right.$, $11^{\circ} 36^{\prime} \mathrm{E}$ ), in Core BK $1 \mathrm{D}$. Comment: sediment studies indicate this level corresponds to max of transgression.

Gif-3453. Kouilou estuary, BK 2 H

$920 \pm 90$

Peaty clay, $10.5 \mathrm{~m}$ below high sea level $\left(4^{\circ} 28^{\prime} 40^{\prime \prime} \mathrm{S}, 11^{\circ} 36^{\prime} \mathrm{E}\right), 50 \mathrm{~m}$ from Core BK 1 D. Comment: corresponds to beginning of new marshy phase.

Gif-3733. Kouilou estuary, BK 2 Y

$6640 \pm 140$

Organic remains in gray clay, $19 \mathrm{~m}$ below high sea level $\left(4^{\circ} 28^{\prime} 30^{\prime \prime} \mathrm{S}\right.$, $\left.11^{\circ} 43^{\prime} 20^{\prime \prime} \mathrm{E}\right)$.

\section{Gif-3735. Kouilou estuary, BK 3 H}

Wood, 14.5 to $17.5 \mathrm{~m}$ below high sea level.

Gif-3734. Kouilou estuary, BK 61

Clayey peat, $26 \mathrm{~m}$ below high sea level $\left(4^{\circ} 28^{\prime} \mathrm{S}, 11^{\circ} 43^{\prime} 20^{\prime \prime} \mathrm{E}\right)$. Comment: corresponds to preceding transgression.

Gif-3452. Kouilou estuary, BK 1 L

Peaty clay, $30 \mathrm{~m}$ below high sea level $\left(4^{\circ} 28^{\prime} 40^{\prime \prime} \mathrm{S}, 11^{\circ} 43^{\prime} 20^{\prime \prime} \mathrm{E}\right)$. Comment: corresponds to preceding transgression.

Gif-2949. "Côte Sauvage", Congo

Modern

Shells (Ostrea denticulata $\mathrm{sp}$ ) from offshore bar, $3 \mathrm{~m}$ above $\mathrm{msl}\left(4^{\circ} 48^{\prime}\right.$ $\left.37^{\prime \prime} \mathrm{S}, 11^{\circ} 51^{\prime} 10^{\prime \prime} \mathrm{E}\right)$. Coll by $\mathrm{G}$ Kouyoumontzakis and subm 1973 by $\mathrm{P}$ Giresse. Comment: oyster shells are not good indicators of sea levels.

Gif-3736. Congo, $1172 \mathrm{C}$

Organic remains in clay at depth $50 \mathrm{~m}$ on continental shelf $\left(5^{\circ} 32^{\prime} \mathrm{S}\right.$, $\left.11^{\circ} 56^{\prime} \mathrm{E}\right)$. Coll and subm 1974 by P Giresse. Comment: corresponds to preceding transgression. 
Gif-3449. Loango, Congo

Organic clay, 0.5m below high sea level $\left(4^{\circ} 39^{\prime} 30^{\prime \prime} \mathrm{S}, 11^{\circ} 47^{\prime} 25^{\prime \prime} \mathrm{E}\right)$. Coll and subm 1974 by P Giresse. Comment: corresponds to slightly lower sea level than at present.

Gif-3450. Songololo R, Congo $\quad 2920 \pm 110$

Organic clay, ca high sea level, from mouth of Songololo R $\left(4^{\circ} 45^{\prime}\right.$ $\left.20^{\prime \prime} \mathrm{S}, 11^{\circ} 51^{\prime} 00^{\prime \prime} \mathrm{E}\right)$. Coll and subm 1974 by P Giresse. Comment: corresponds to slightly lower sea level than at present.

\section{Gif-2564. Congo, P G $596 \quad 11,980 \pm 250$}

Amphistegines from sediment dredged from Congo continental shelf $\left(4^{\circ} 14^{\prime} 5^{\prime \prime} \mathrm{S}, 11^{\circ} 04^{\prime} 6^{\prime \prime} \mathrm{E}\right)$, at depth $112 \mathrm{~m}$. Subm 1972 by P Giresse. Comment: Amphistegines are large foraminifera living at depth of $55 \mathrm{~m}$ or less. It can be deduced that sea level was ca $-60 \mathrm{~m}$ (Delibrias, Giresse, and Kouyoumontzakis, 1973).

\section{Gif-3479. Baia Forta, Angola}

$3400 \pm 100$

Shells (Arca senilis) from midden at $20 \mathrm{~m}$ above msl $\left(12^{\circ} 40^{\prime} \mathrm{S}, 13^{\circ} 12^{\prime}\right.$ E), S W Benguela. Coll and subm 1974 by G Kouyoumontzakis, Lab Geol, Univ Brazzaville, Congo. Comment: corresponds to max of Nouakahottian transgression (Giresse, Kouyoumontzakis, and Delibrias, 1976).

\section{Gif-3477. Baia Azul, Angola $\quad 20,600 \pm 400$}

Shells (Arca senilis), at 10 to $12 \mathrm{~m}$ above msl $\left(12^{\circ} 38^{\prime} \mathrm{S}, 13^{\circ} 15^{\prime} \mathrm{E}\right)$. Coll and subm 1974 by $G$ Kouyoumontzakis. Comment: aberrant date that cannot be explained by recrystallization of shells, which were $100 \%$ aragonite; probably mixing of shells from different levels occurred.

\section{Gif-2941. Praia Amelia, Mossamedes, Angola GKAN 10}

$18,200 \pm 400$

Beach deposit with Strombusbubonius, Arca senilis, Conus spp, 1.5 to $2.5 \mathrm{~m}$ above msl, Praia Amelia $\left(15^{\circ} 12^{\prime} \mathrm{S}, 12^{\circ} 06^{\prime} \mathrm{E}\right), \mathrm{S}$ Mossamedes. Coll by $\mathrm{G}$ Kouyoumontzakis and subm 1973 by $\mathrm{P}$ Giresse. Comment: date disagrees with known sea level at 18,000 BP; probably mixing of ancient and more recent shells occurred.

\section{Gif-3732. Angola, GKAN 54}

Marine shells from uplifted level at $20 \mathrm{~m}$ above msl $\left(12^{\circ} 35^{\prime} \mathrm{S}, 13^{\circ}\right.$ $25^{\prime} \mathrm{E}$ ). Coll and subm 1975 by $\mathrm{P}$ Giresse. Comment: corresponds to ancient high sea level, probably from Ouljian transgression.

Gif-2945. Ponta da Giraul, N Mossamedes, Angola $\quad 3430 \pm 100$

Broken shell in raised beach, at $5 \mathrm{~m}$ above msl $\left(15^{\circ} 06^{\prime} \mathrm{S}, 12^{\circ} 07^{\prime} \mathrm{E}\right)$. Coll and subm 1973 by $\mathrm{P}$ Giresse. Comment: dates one of raised beaches observed in S Angola, which disappear towards N Angola.

Gif-2946. Praia des Conchas, N Mossamedes, Angola $3040 \pm 100$

Serpulides tubicoles lumachelle, 2 to $4 \mathrm{~m}$ thick at $3 \mathrm{~m}$ above $\mathrm{msl}\left(15^{\circ}\right.$ $05^{\prime} \mathrm{S}, 12^{\circ} 08^{\prime} \mathrm{E}$ ). Coll and subm 1973 by P Giresse. Comment: same as for Gif-2945 (Giresse, Kouyoumontzakis, and Delibrias, 1973). 
Gif-2947. Baia Forta, Angola

Shell interbedded in coarse sand, 2 to $3 \mathrm{~m}$ above msl $\left(12^{\circ} 37^{\prime} \mathrm{S}, 13^{\circ}\right.$ 12' E). Coll and subm 1973 by $\mathrm{P}$ Giresse. Comment: corresponds to Ouljian transgression.

Gif-3230. Praia Amelia, Angola

$1620 \pm 80$

Marine shells, at $5 \pm 2 \mathrm{~m}$ above msl $\left(15^{\circ} 12^{\prime} \mathrm{S}, 12^{\circ} 06^{\prime} \mathrm{E}\right)$. Coll and subm 1974 by $G$ Kouyoumontzakis.

\section{Gif-3231. Paia des Conchas, Angola}

Modern

Shell remains, at 2 to $3 \mathrm{~m}$ above msl $\left(15^{\circ} 05^{\prime} \mathrm{S}, 12^{\circ} 08^{\prime} \mathrm{E}\right)$. Coll and subm 1974 by $\mathrm{G}$ Kouyoumontzakis. Comment: corresponds to high sea level.

Gif-3478. Baia du Pipas, Angola

Marine shells, at $15 \mathrm{~m}$ above msl $\left(15^{\circ} 07^{\prime} \mathrm{S}, 12^{\circ} 12^{\prime} \mathrm{E}\right)$. Coll and subm 1974 by $\mathrm{G}$ Kouyoumontzakis. Comment: corresponds to ancient high sea level, probably from Ouljian transgression.

\section{F. East Africa and Madagascar}

\section{Gif-2559. N coast Ghoubbet al Karab, W Djibouti,} Ethiopia

$27,600 \pm 1500$

Shells sampled at alt $10 \mathrm{~m}$, from tectonically uplifted fringe reef, $60 \mathrm{~m}$ high, N coast of Ghoubbat al Karab $\left(11^{\circ} 32^{\prime} \mathrm{N}, 42^{\circ} 32^{\prime} \mathrm{E}\right)$, Tadjoura Gulf. Coll and subm 1972 by L Stieltjes, BRGM, La Reunion. Comment: inconsistent with uplift rates of this littoral zone deduced from ${ }^{430} \mathrm{Th} /{ }^{234} \mathrm{U}$ dates on corals (Faure, Hoang, and Lalou, 1980). Results from light contamination of shells by recent carbon. Date must be taken as min (Stieltjes, 1973).

\section{Gif-2558. N coast Ghoubbet al Karab, W Djibouti, Ethiopia}

Shells from lacustrine deposit assoc with max SE extension of Lake Asal $\left(11^{\circ} 35^{\prime} \mathrm{N}, 42^{\circ} 30^{\prime} \mathrm{E}\right)$, Tadjoura Gulf. Coll and subm 1972 by $\mathrm{L}$ Stieljes.

Gif-2554. Ghoubbet al Karab, Afar, Ethiopia

$7400 \pm 110$

Shells (Melania) from lacustrine sediments under basaltic flow, in open fissure in lava $\left(11^{\circ} 30^{\prime} \mathrm{N}, 42^{\circ} 30^{\prime} \mathrm{E}\right)$. Coll and subm 1972 by $\mathrm{M}$ Marinelli, Inst Min Petrog, Pisa. Comment: eolian sediment is interbedded between lava and lacustrine deposit. Date is max for basaltic flow, one of last of Ghoubbet, which is probably younger than $7000 \mathrm{BP}$. With width of fissure at ca $40 \mathrm{~m}$ it is possible to calculate min spreading rate of ca $1 \mathrm{~cm} / \mathrm{yr}$ for Asal ridge (Delibrias, Marinelli, and Stieljes, 1974).

\section{Fort-Dauphin series, Madagascar}

Fossil vermetid shells from Fort-Dauphin region $\left(25^{\circ} 01^{\prime} \mathrm{S}, 47^{\circ} 00^{\prime}\right.$ E). Coll and subm 1973 by J Laborel, Sta marine d'Endoume, Marseille. Alt given above biol level of living vermets. 
Gif-2838. Fort Dauphin 1

Fossil formation, $2 \pm 0.5 \mathrm{~m}$.

Gif-2839. Fort-Dauphin 3

$1920 \pm 90$

Fossil formation, $2.5 \pm 0.5 \mathrm{~m}$.

Gif-2449. Sambava, Madagascar

$2020 \pm 90$

Shells in sandy bar, at approx msl $\left(14^{\circ} 16^{\prime} \mathrm{S}, 50^{\circ} 10^{\prime} \mathrm{E}\right)$, NE Madagascar. Coll and subm 1973 by R Battistini, Dept Géog, Univ d'Orléans.

General Comment for Madagascar samples: evidence of high sea levels during Holocene is observed along coast of Madagascar.

\section{Tulear series, Madagascar}

Littoral samples from Tulear region, SW Madagascar. Coll and subm 1972 by M Pichon, Sta marine d'Endoume, Marseille.

Gif-2520. Tulear, MP/D 29

$1870 \pm 100$

Organogenic sandstone of recent formation dredged from depth 40 to $45 \mathrm{~m}$ near Tulear $\left(23^{\circ} 24^{\prime} 9^{\prime \prime} \mathrm{S}, 43^{\circ} 38^{\prime} 4^{\prime \prime} \mathrm{E}\right)$, SW Madagascar.

Gif-2519. Tulear, 4-MP/A1

$4800 \pm 130$

Thick pieces of Aepyornis egg shells, coll from yellow dune largely consisting of quartz sands.

Gif-2521. Tulear, 4-MP/A2

$1530 \pm 110$

Thin pieces of Aepyornis egg shells, coll from white dune largely consisting of organogenic limestone, near Tulear $\left(23^{\circ} 17^{\prime} 8^{\prime \prime} \mathrm{S}, 43^{\circ} 38^{\prime} 5^{\prime \prime} \mathrm{E}\right)$. General Comment: eggs date littoral dune systems. Organogenic limestone in white dunes comes from coral indicating coral littoral formation between 4800 and $1530 \mathrm{BP}$, existence of which is proven by dredged sample. Aepyornis is now extinct in Madagascar; birds were still extant when first Europeans arrived. Thinness of shells (Gif-2521) is considered evidence of degenerative stage of $\mathrm{sp}$ (M P).

Gif-2391. Vavatenina region, Madagascar IFB13 $2270 \pm 90$

B horizon from podzol lying on alluvial terrace $\left(17^{\circ} 27^{\prime} \mathrm{S}, 49^{\circ} 20^{\prime} \mathrm{E}\right)$. Coll and subm 1971 by $F$ Bourgeat, Ecole Sup Nat Agron, Paris. Comment: date is upper limit for end of terrace formation.

\section{G. Pacific Coasts}

\section{Reef Island series, Bank Island Group, New Hebrides}

Samples of coral and shell from Reef I. (13 $37^{\prime} \mathrm{S}, 167^{\circ} 32^{\prime} \mathrm{E}$ ), Bank I. Group, New Hebrides. Coll and subm 1972 by A Guilcher, Fac Lettres Sci Humaines, Brest.

Gif-2535. Reef 2

$$
4320 \pm 80
$$

Coral, E Wosou, mid-tide level.

Gif-2536. Reef 3

$5980 \pm 90$

Coral, NE Wosou, mid-tide level. 
Gif-2531. Reef 8

$4570 \pm 80$

Shell (Tridacne), SW Wosou, just above mid-tide level.

Gif-2532. Reef 17

$4150 \pm 80$

Shell (Pemphis), NW Reef I., mid-tide level.

Gif-2534. Reef 18

$4970 \pm 80$

Shell (Tridacne), NW Reef I., same level as Reef 17.

Gif-2533. Reef 45

$6640 \pm 100$

Coral, S Rowa I., mean high-tide level.

\section{Gif-2624. Reef 44}

$3270 \pm 100$

Coral, S Rowa I., filling of pipe.

\section{Gif-2625. Reef 43}

$20,600 \pm 320$

Coral, from outer edge of reef Rowa $\mathrm{I}$.

General Comment: dates indicate that old reef was formed during Holocene when sea level was $0.5 \mathrm{~m}$ to $1.5 \mathrm{~m}$ higher than at present. Seismicity of archipelago is high; thus it is impossible to know that alt of corals is due only to sea-level variations. All dated corals were in growth position except for Gif-2625, which results from mixture with older corals. Reef I. probably had shallow Pleistocene basement (Guilcher, 1974).

Gif-3566. Farquhar I., Seychelles

$3640 \pm 100$

Sample of coralline limestone $2.5 \mathrm{~m}$ above sea level, from rim of Farquhar atoll $\left(10^{\circ} \mathrm{S}, 51^{\circ} \mathrm{E}\right)$. Coll and subm 1975 by R Battistini. Comment: two U-Th dates for same coral sample, $4700 \pm 600$ and $3100 \pm 700$ BP (Battistini, Delibrias, and Laborel, 1976), show good correlation obtainable betwcen ${ }^{14} \mathrm{C}$ and U-Th dates for young coral samples.

\section{New Caledonia series}

Peat samples from mangrove peat bogs on $\mathrm{W}$ coast of New Caledonia. Coll and subm 1973 by F Baltzer, Fac Sci Orsay, Essonne.

Gif-2929. Nessadiou $10 \mathrm{E}$ $5930 \pm 120$

Peat $\left(21^{\circ} 38^{\prime} \mathrm{S}, 168^{\circ} 28^{\prime} 30^{\prime \prime} \mathrm{E}\right)$.

Gif-2930. Marais de Mara, 4-1 $5330 \pm 120$ Peat (21 $\left.45^{\prime} \mathrm{S}, 165^{\circ} 41^{\prime} 30^{\prime \prime} \mathrm{E}\right)$.

Gif-2932. Boulouhari, 104 Peat $\left(21^{\circ} 55^{\prime} \mathrm{S}, 165^{\circ} 56^{\prime} \mathrm{E}\right)$.

General Comment: dates stages of regression of sea during Holocene transgression. 
Gif-2392. La Reunion, LRZ443

$1330 \pm 90$

B horizon from podzol $\left(21^{\circ} 6^{\prime} 13^{\prime \prime} \mathrm{S}, 55^{\circ} 21^{\prime} 26^{\prime \prime} \mathrm{E}\right), 16$ to $26 \mathrm{~cm}$ below surface, at alt $1650 \mathrm{~m}$. Coll and subm 1971 by C Zebrowski, ORSTOM, Paris.

Gif-2393. La Reunion, LRZ463

$2100 \pm 90$

B horizon from podzol $\left(21^{\circ} 10^{\prime} 53^{\prime \prime} \mathrm{S}, 55^{\circ} 37^{\prime} 50^{\prime \prime}\right)$, at depth 80 to $95 \mathrm{~cm}$ from under volcanic ejecta. Coll and subm 1971 by C Zebrowski. Comment: dates upper limit of volcanic event.

\section{Vietnam series}

During Holocene transgression, sea submerged present coastal plains in Vietnam, especially Mekong Delta. Much archaeol and geol evidence of ancient coastline exists and indicates higher sea levels than at present (Fontaine and Delibrias, 1974). Coll and subm 1972-1974 by H Fontaine, Serv Geol, Saigon.

Gif-2647. Ben-Dô $3000 \pm 110$

Organic remains in ceramic debris, from archaeol site in highlands Mekong Delta $\left(10^{\circ} 52^{\prime} \mathrm{N}, 106^{\circ} 51^{\prime} \mathrm{E}\right)$. As lowlands were probably being submerged or were marshy, prehistoric people settled areas bordering Mekong Delta.

Gif-2394. Rach-Nui

$2400 \pm 100$

Shells in kitchen refuse, from site believed to be earliest settlement in lowlands of Mekong Delta $\left(10^{\circ} 33^{\prime} \mathrm{N}, 106^{\circ} 40^{\prime} 30^{\prime \prime} \mathrm{E}\right)$.

Gif-2648. Nha-Trang, Ire Island

$3090 \pm 110$

Emerged coral from Nha-Trang, Ire I.

Gif-2474. Ca-Na

$2490 \pm 100$

Coral from "Terrace" $\left(11^{\circ} 20^{\prime} \mathrm{N}, 108^{\circ} 5 \mathrm{l}^{\prime} \mathrm{E}\right)$, ca $2 \mathrm{~m}$ above msl. "Terraces" are accumulation of cemented coral debris and shells on high beach.

Gif-2475. Ca-Na

$$
4200 \pm 110
$$
msl.

Coral from "new terrace" (11 $\left.20^{\prime} \mathrm{N}, 108^{\circ} 52^{\prime} 30^{\prime \prime} \mathrm{E}\right)$, ca $2 \mathrm{~m}$ above

Gif-2476. Ca-Na

$900 \pm 90$

Shells and coral from "terrace", Dông-Hai $\left(12^{\circ} 30^{\prime} \mathrm{N}, 109^{\circ} 18^{\prime} \mathrm{E}\right)$, $1.2 \mathrm{~m}$ above msl.

\section{Bahia series}

H. Brazil

Shelly sandstone from ancient littoral zone $1.5 \mathrm{~m}$ above msl, near Bahia $\left(13^{\circ} \mathrm{S}, 38^{\circ} \mathrm{W}\right)$. Coll and subm 1971 by $\mathrm{T}$ Da Silva, Univ Bahia, Salvador, Brazil.

Gif-2149. Bahia 1

$2150 \pm 100$

Calcareous cement of sandstone. 
Gif-2150. Bahia 2

$3780 \pm 130$

Shell extracted from sandstone. Comment: discrepancy between two ages means either successive recrystallization of cement results in younger dates, or older shells were cemented into sandstone.

Gif-2957. Porto de Galinhas, Pernambuco

$980 \pm 90$

Sandstone with Halimeda sp on coral slab, $0.5 \mathrm{~m}$ above msl. Coll and subm 1973 by J Laborel. Comment: validity of date on carbonate from sandstone is impossible to evaluate because of possible incorporation of ancient carbonate; however, date is acceptable.

\section{Quebec city area series}

\section{Canada}

Peat bogs from Quebec city area. Coll and subm 1970 by P Richard, Univ Quebec, Chicontini, Canada (Richard, 1971).

Gif-1756. Saint-Jean, Ile d'Orléans, J!

$1180 \pm 100$

Peat from depth 70 to $85 \mathrm{~cm}$ from Saint-Jean bog, $4.2 \mathrm{~m}$ thick, on Orléans I., in St Lawrence R, E Quebec city $\left(47^{\circ} 56^{\prime} \mathrm{N}, 71^{\circ} 56^{\prime} \mathrm{W}\right)$. Comment: pollen analysis indicates Tsuga canadensis max.

Gif-1757. Saint-Jean, Ile d'Orléans, J2

Peat from depth 270 to $285 \mathrm{~cm}$ from same peat bog. Comment: pollen analysis indicates Fagus increase; Acer forest prevails.

Gif-1758. Saint-Jean, Ile d'Orléans, J3

$6100 \pm 160$

Peat from depth 320 to $33 \check{\mathrm{cm}}$ from same peat bog. Comment: pollen analysis indicates Pinus strobus max, Acer, Tilia, and Fagus appearance. End of Betula forest.

Gif-1759. Saint-Raymond, Portneuf, RAY $1 \quad 3550 \pm 120$

Peat from depth 270 to $285 \mathrm{~cm}$ from Saint-Raymond peat bog, Portneuf $\left(46^{\circ} 53^{\prime} \mathrm{N}, 71^{\circ} 48^{\prime} \mathrm{W}\right)$. Comment: high pollen frequency level, not correlated with J3 level as expected. Bottom of RAY profile dated to $7970 \pm 140$ вр (GSC-1400).

\section{ARCHAEOLOGIC SAMPLIS}

\section{Wissant series, Nord}

\section{A. France}

Littoral archaeol sites of interest to both archaeol and sea-level studies at Wissant $\left(50^{\circ} 53^{\prime} \mathrm{N}, 0^{\circ} 42^{\prime} \mathrm{E}\right)$, Pas-de-Calais. Samples coll and subm 1969-1972 by H Mariette, Samer, Pas-de-Calais.

Gif-1598. Wissant 1

$4520 \pm 120$

Shell (Cardium) from brackish clay level with scrobiculaires, on foreshore at msl. 
Gif-1768. Wissant 2

$3610 \pm 110$

Sandy peat lying on sand, at top of beach $2.7 \mathrm{~m}$ above msl. Comment: considering tidal range of $7 \mathrm{~m}$, date corresponds to lowering of $0.8 \mathrm{~m}$ below msl; agrees with assoc of Neolithic industry.

Gif-2678. Wissant 3

$1840 \pm 90$

Charcoal in clayey level in sand cliff; assoc with ceramics difficult to date by achaeol estimates.

\section{Hardelot series, Pas-de-Calais}

Samples from archaeol littoral site, interbedded in sand on beach $\left(50^{\circ} 36^{\prime} \mathrm{N}, 0^{\circ} 45^{\prime} \mathrm{E}\right)$. Coll and subm $1969-1970$ by $\mathrm{H}$ Mariette.

Gif-1770. Hardelot 1

$2740 \pm 100$

Humic sand, $7 \mathrm{~m}$ above msl. Comment: agrees with assoc of 1st Iron age industry.

Gif-1600. Hardelot 3

$2950 \pm 110$

Sandy peat, $6.5 \mathrm{~m}$ above msl.

Gif-1599. Hardelot 2

$3000 \pm 110$

Sandy peat, $6 \mathrm{~m}$ above $\mathrm{msl}$.

Gif-1769. Hardelot 4

$2930 \pm 100$

Peaty sand, $4 \mathrm{~m}$ above msl. Comment: presence of potsherds of Bronze age should date sample some hundreds of years older.

Gif-2557. Wimereux, Pas-de-Calais

$3620 \pm 110$

Wood in peat containing tree trunks, at top of beach $3 \mathrm{~m}$ above msl $\left(56^{\circ} 43^{\prime} \mathrm{N}, 0^{\circ} 81^{\prime} \mathrm{E}\right)$. Coll and subm 1971 by $\mathrm{H}$ Mariette. Comment: peat layer was between 2 levels of Neolithic and La Tène periods. Date is consistent with this archaeol evidence.

Gif-2677. Etaples, Pas-de-Calais

$3420 \pm 100$

Charcoal from site Etaples $\left(50^{\circ} 31^{\prime} \mathrm{N}, \mathrm{I}^{\circ} 38^{\prime} \mathrm{E}\right)$. Coll 1956 and subm 1972 by H Mariette. Comment: date agrees with Campaniforme ceramics assoc, similar to "Bell beaker" types from Netherlands (Mariette, 1959).

\section{Noisy-sur-Ecole series, Seine et Marne}

Charcoal from collective tomb of SOM (Seine-Oise-Marne) Neolithic type $\left(48^{\circ} 21^{\prime} \mathrm{N}, 2^{\circ} 27^{\prime} \mathrm{E}\right)$. Coll and subm 1971 by M Brézillon, Antiquités Préhist, Paris.

Gif-2241. Noisy-sur-Ecole, A

$4480 \pm 110$

Charcoal coll in rectangular room at depth $1 \mathrm{~m}$, assoc with two human skeletons.

Gif-2242. Noisy-sur-Ecole, B

$4530 \pm 110$

Charcoal mixed with human bones around well, in 2nd elliptical room. 
Gif-2243. Noisy-sur-Ecole, $C$

$2970 \pm 100$

Charcoal in well at depth 3 to $4 \mathrm{~m}$, under remains of domestic fauna, atypical stone industry, and pottery of Hallstatt period.

General Comment: Gif-2241 and -2242 agree with archaeol evidence for SOM Neolithic civilization. Gif-2243 was expected to be $500 \mathrm{yr}$ younger.

Gif-2365. Cannes-Ecluse, Seine et Marne

$1970 \pm 70$

Charcoal from Late Bronze age site at depth $70 \mathrm{~cm}$, "Les Bagneux" $\left(48^{\circ} 22^{\prime} \mathrm{N}, 3^{\circ} 0 \breve{3}^{\prime} \mathrm{E}\right)$. Coll by C Mordant and subm 1971 by M Brézillon. Comment: dates conflict with archaeol evidence; no explanation offered.

Gif-2366. Misy, Seine et Marne

$540 \pm 60$

Charcoal from Middle Bronze age site at depth $40 \mathrm{~cm}$, "Bois des

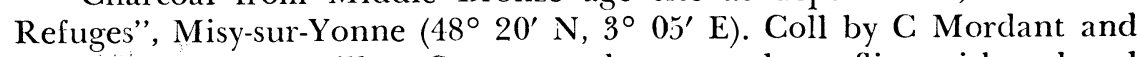
subm $197 \mathrm{l}$ by M Brézillon. Comment: date strongly conflicts with archaeol evidence.

Gif-2721. Sèvres, Hauts de Seine

$5190 \pm 130$

Charcoal from gallery for flint quarrying from chalk formation $\left(48^{\circ}\right.$ $20^{\prime} \mathrm{N}, 2^{\circ} 27^{\prime} \mathrm{E}$ ). Coll by J L Maire and subm 1972 by M Brézillon. Comment: date agrees with expected age.

Gif-2723. Germigny-L'Evêque, Seine et Marne $\quad 3970 \pm 120$

Charcoal from hearth in collective tomb of SOM Neolithic type $\left(48^{\circ}\right.$ $\left.59^{\prime} \mathrm{N}, 2^{\circ} 56^{\prime} \mathrm{E}\right)$. Coll by J Tarrête and subm 1973 by M Brézillon. Comment: date agrees with expected age.

Gif-2169. Loisy-en-Brie, Marne

$3690 \pm 100$

Bones from collective tomb in hypogeum of Loisy-en-Brie $\left(48^{\circ} 54^{\prime} \mathrm{N}\right.$, $4^{\circ} \mathrm{E}$ ), in rich archaeol context of SOM culture. Subm by M Joffroy, Saint-Germain-en-Laye. Comment: date agrees well with archaeol data.

\section{Gif-1604. Le Rozel, Manche}

$17,000 \pm 700$

Organic horizon in Upper Paleolithic rockshelter in natural fault in vertical cliff, at Pointe du Rozel $\left(49^{\circ} 28^{\prime} \mathrm{N}, 1^{\circ} 50^{\prime} 30^{\prime \prime} \mathrm{W}\right)$, coast of Cotentin. Coll and subm 1970 by F Scuvée, Digosville, Manche. Comment: dated as expected although very poor industry of site did not allow accurate archaeol interpretation.

Gif-2291. Gaignog Island, Landeda, Finistère

Charcoal from Dark age site in Gaignog I. $\left(48^{\circ} 35^{\prime} \mathrm{N}, 4^{\circ} 35^{\prime} \mathrm{W}\right)$. Coll and subm 1971 by P R Giot, Fac des Sci, Rennes. Comment: charcoal is related to oldest enclosure of site.

\section{Gif-2293. Kerascoët, Saint Segal, Finistère}

$970 \pm 90$

Charcoal from surrounding wall of camp of Kerascoët $\left(48^{\circ} 15^{\prime} \mathrm{N}, 4^{\circ}\right.$ $3^{\prime}$ W). Coll by L E Gall and subm 1971 by R Sanquer, Fac Lettres, Brest. Comment: gives early medieval date for this type of circular camp. 
Saint-Urnel, Plomeur series, Finistère

Littoral necropolis of Saint-Urnel, Plomeur $\left(47^{\circ} 55^{\prime} \mathrm{N}, 4^{\circ} 20^{\prime} \mathrm{W}\right)$ is famous cemetery. Its numerous skeletons, interbedded in dune were well studied to determine origin of settlement in Brittany. Lying on Bronze age soil, it was first attributed to Iron age (Giot and Cogné, 1951). Samples coll and subm 1971-1976 by P R Giot.

Gif-2296. Saint-Urnel, S U 1

$970 \pm 90$

Human bones from upper level.

Gif-2681. Saint-Urnel, S U 2

$980 \pm 90$

Human bones from same level as Gif-2296.

Gif-3079. Saint-Urnel, S U 1973

$900 \pm 90$

Charcoal from furnace used in bronze metallurgy for casting bells.

Gif-3584. Saint-Urnel, S U 74-109

$1630 \pm 90$

Human bones from one of most ancient sepultures.

Gif-3745. Saint-Urnel, S U 75, 7.8.9/CD

$1300 \pm 90$

Charcoal from oratory in center of necropolis.

Gif-3795. Saint-Urnel, S U 74-61

$1400 \pm 90$

Human bones from ancient sepulture.

Gif-4071. Saint-Urnel, S U 74 151B

$1310 \pm 90$

Human bones under post hole of oratory.

General Comment: dates necropolis to Dark age and High Middle age which is also period of arrival of Breton people in region (Giot and Monnier, 1977; 1978).

Gif-2326. Saint-Servan, Ille et Vilaine

$1860 \pm 90$

Wood from worked piece of machinery engine (Roman pump) from big tank embedded into gneiss rock, at Saint-Servan-Saint Malo $\left(48^{\circ} 38^{\prime}\right.$ $\mathrm{N}, 2^{\circ} 02^{\prime} \mathrm{W}$ ) (Langouët and Meury, 1973). Coll and subm 1971 by L Langouët, Fac Sci, Rennes. Comment: date agrees with expected age.

\section{Gif-2327. Kerbat-Plouyé, Finistère}

$2380 \pm 70$

Charcoal from Iron age souterrain (underground tunnel) $\left(48^{\circ} 19^{\prime} \mathrm{N}\right.$, $3^{\circ} 43^{\prime}$ W). Coll and subm 1971 by C T Le Roux, Fac Sci, Rennes. Comment: fits with archaeol evidence.

\section{Kerlande series, Brandivy, Finistère}

Charcoal from late Bronze age site at Kerlande $\left(47^{\circ} 46^{\prime} \mathrm{N}, 2^{\circ} 54^{\prime} \mathrm{W}\right)$. Coll by J Lecornec and subm 1971 by P R Giot.

Gif-2379. Kerlande, $\mathrm{C}^{\prime} \mathbf{2 - 2 7}$

$$
860 \pm 90
$$

Charcoal.

Gif-2331. Kerlande, 1

$1800 \pm 90$

Charcoal. 
318 Georgette Delibrias, Marie-Thérèse Guillier, and Jacques Labeyrie

Gif-2378. Kerlande, $A^{\prime} 4-90 \quad 2820 \pm 100$

Charcoal.

General Comment: Gif-2379 and -2331 date some recent periods of occupation of this enclosed site of Late Bronze age which were not revealed during excavation. Gif-2378 agrees with expected age (Lecornec, 1973).

\section{Kerviny barrow series, Poullan, Finistère}

Charcoal from Early Bronze age barrow (48 $\left.3^{\circ} 14^{\prime \prime} \mathrm{N}, 4^{\circ} 22^{\prime} 51^{\prime \prime} \mathrm{W}\right)$. Coll and subm 1971 by J Briard, Fac Sci, Rennes.

\section{Gif-2374. Kerviny 2}

$1760 \pm 90$

Charcoal from hearth.

Gif-2480. Kerviny 1

Modern

Charcoal.

Gif-2481. Kerviny 3

$3510 \pm 100$

Charcoal.

General Comment: only Gif-2481 gives expected age of barrow; Gif-2374 and -2481 indicate later re-occupations (Briard, 1972).

Kerviny settlement series, Poullan, Finistère

Charcoal from proto-historic enclosure beside barrow at Kerviny. Coll and subm 1971 by J Briard.
Gif-2377. Kerviny 6
$940 \pm 90$
Charcoal.
Gif-2375. Kerviny 4
$1430 \pm 90$
Charcoal.
Gif-2376. Kerviny 5

Charcoal.

General Comment: dates indicate long and continual settlement of site.

Gif-2380. Meilars, Penguilly, Finistère

$2500 \pm 100$

Charcoal from barrow of Penguilly $\left(48^{\circ} 4^{\prime} \mathrm{N}, 4^{\circ} 26^{\prime} \mathrm{W}\right)$. Coll and subm by J Briard. Comment: typical monument of Bronze age (Briard and Penziat, 1972).

Gif-2381. Plourin-Ploudalmezeau, Milinigou, Finistère $950 \pm 90$

Charcoal from Bronze age sepulture of Milinigou $\left(48^{\circ} 30^{\prime} 25^{\prime \prime} \mathrm{N}, 4^{\circ}\right.$ $\left.39^{\prime} 37^{\prime \prime} \mathrm{W}\right)$. Coll and subm 1971 by J Briard. Comment: dates re-use of monument.

Gif-2382. Plonéis, Penanguer, Finistère $2380 \pm 100$

Wood from piles in ancient marsh $\left(48^{\circ} 03^{\prime} \mathrm{N}, 4^{\circ} 13^{\prime} \mathrm{W}\right)$. Coll by $\mathrm{J}$ Garrec and subm 1971 by P R Giot. 
Charcoal from hearth under Roman bldg near Lilia, Plougerneau $\left(48^{\circ} 38^{\prime} \mathrm{N}, 4^{\circ} 34^{\prime} \mathrm{W}\right)$. Coll and subm 1971 by R Sanquer, Fac Sci, Brest. Comment: slightly older than expected.

\section{Ploudaniel series, Finistère}

Charcoal from tombstones in Bronze age barrow at Kerno, Ploudaniel $\left(48^{\circ} 33^{\prime} 24^{\prime \prime} \mathrm{N}, 4^{\circ} 19^{\prime} 22^{\prime \prime} \mathrm{W}\right)$. Coll and subm 1971 by J Briard.

\section{Gif-2292. Ploudaniel, Kerno $A \quad 2830 \pm 110$ \\ Charcoal. \\ Gif-2421. Ploudaniel, Kerno B \\ $3450 \pm 100$}

Charcoal.

General Comment: Kerno B gives expected date for this Bronze age monument; charcoal from Kerno $\mathrm{A}$ is probably contaminated (Briard and Gouletquer, 1972).

Gif-2471. Grande Brière, Loire Atlantique

$4680 \pm 110$

Human bones in sepulture at depth $0.9 \mathrm{~m}$ in peat bog of La Grande Brière $\left(47^{\circ} 08^{\prime} \mathrm{N}, 1^{\circ} 96^{\prime} \mathrm{W}\right)$. Coll by $\mathrm{G}$ Bellancourt and subm 1972 by J L'Helgouach, Antiquités Prehist Pays Loire, Nantes. Comment: date is similar to those of peat and wood coll elsewhere in peat bog, dated by Sa-39-41-42-46:4630, 4480, 4100, 4260 вр (R, 1964, v 6, p 234).

\section{Gif.2472. Pleneuf, Nantois, Côtes du Nord $\geqslant \mathbf{4 0 , 0 0 0}$}

Peaty silt in littoral cutting beneath $8 \mathrm{~m}$ of sand, soils, silt, loess, and solifluction formations ( $\left.48^{\circ} 36^{\prime} \mathrm{N}, 2^{\circ} 51^{\prime} 20^{\prime \prime} \mathrm{W}\right)$. Coll and subm 1972 by J L Monnier, Fac Sci, Rennes. Comment: consistent with geol estimate which places soil in Eemian interglacial.

Gif-2473. Kerjouanno, Arzon, Morbihan

$2570 \pm 100$

Charcoal in old soil under dune of Kerjouanno $\left(47^{\circ} 32^{\prime} \mathrm{N}, 2^{\circ} 52^{\prime} \mathrm{W}\right)$. Coll 1970 by P L Gouletquer and subm 1972 by P R Giot. Comment: unrelated to Mesolithic site found under same dune.

Gif-2631. Crann, La Forêt-Landerneau, Finistère $\quad 7580 \pm 110$

Charcoal from quartzite chipping workshop in quarry $\left(48^{\circ} 25^{\prime} \mathrm{N}, 4^{\circ}\right.$ $21^{\prime}$ W). Coll by P R Giot, B Hallegouët and J L Monnier and subm 1972 by P R Giot. Comment: date for crude lithic industry.

Gif-2685. Les Touches, Mohon, Morbihan $\quad 2300 \pm 100$

Charcoal from Iron age souterrain $\left(49^{\circ} 35^{\prime} \mathrm{N}, 2^{\circ} 32^{\prime} \mathrm{W}\right)$. Coll by $\mathrm{J}$ Lecornec and subm 1972 by P R Giot. Comment: date agrees with archaeol data.

St int-Jude, Bourbriac series, Côtes du Nord

Charcoal from different parts of barrow of Saint-Jude, at Bourbriac $\left(48^{\circ} 27^{\prime} 18^{\prime \prime} \mathrm{N}, 3^{\circ} 8^{\prime} 24^{\prime \prime} \mathrm{W}\right)$. Coll and subm 1972 by J Briard (Briard $e t$ al, 1977). 
Charcoal.

Gif-2687. Saint-Jude, B 2

$3870 \pm 100$

Charcoal.

Gif-2688. Saint-Jude, B 3

$3760 \pm 100$

Charcoal.

General Comment: dates show that this early Bronze age monument was built in one phase.

Gif-2689. Pont-Croix, Finistère

$1900 \pm 100$

Charcoal from Romano-Gallic villa, at Kervenennec, Pont-Croix $\left(48^{\circ}\right.$ $\left.2^{\prime} \mathrm{N}, 4^{\circ} 33^{\prime} \mathrm{W}\right)$. Coll and subm 1972 by R Sanquer. Comment: appears slightly older than expected from ceramics and coins found on site. Date was estimated at ca AD 250-275.

Gif-2765. Longeville, Vendée

$2610 \pm 110$

Charcoal from Hearth A IV with four whole pots, ca $1 \mathrm{~m}$ below high sea level, on beach of Longeville $\left(46^{\circ} 24^{\prime} 15^{\prime \prime} \mathrm{N}, 1^{\circ} 30^{\prime} 12^{\prime \prime} \mathrm{W}\right)$. Coll and subm 1973 by J L'Helgouach. Comment: date is appropriate for early Iron age in Vendée; shows sea level was lower than at present.

Gif-2766. Athée, "la Petite Gaudinière", Mayenne $\quad 390 \pm 90$

Wood from ancient gold mine at "la Petite Gaudinière" $\left(47^{\circ} 52^{\prime} \mathrm{N}\right.$, $\left.0^{\circ} 54^{\prime} 20^{\prime \prime} \mathrm{W}\right)$. Coll and subm 1973 by J L'Helgouach. Comment: date indicates recent exploitation of gold in Mayenne.

\section{Plussulien series, Côtes du Nord}

Charcoal from Neolithic factory for roughed-out axes of dolerites at Seledin, Plussulien $\left(48^{\circ} 13^{\prime} \mathrm{N}, 3^{\circ} 03^{\prime} \mathrm{W}\right)$. Dates extend series from site, lst series pub in R, 1974, v 16, p 17; Delibrias and Le Roux (1975). Coll and subm 1972-1976 by C T Le Roux.

Gif-2682. Plussulien, N, SE

$4940 \pm 120$

Charcoal.

Gif-2683. Plussulien, N, SW

$4350 \pm 120$

Charcoal.

Gif-2684. Plussulien, 02, NE

$4940 \pm 120$

Charcoal.

Gif-3098. Plussulien, 03, NW

$4420 \pm 110$

Charcoal.

Gif-4079. Plussulien, 1976

$4730 \pm 110$

Charcoal.

General Comment: dates and stratigraphic study provide coherent history of site. Three important phases of activity were id and dated from 4350 to 5270 вP (not calibrated). 


\section{Gif-3550. Piedmont, Port des Barques, Charente} Maritime

$4290 \pm 110$

Wood from refuse pit on foreshore at Piedmont $\left(45^{\circ} 57^{\prime} \mathrm{N}, 1^{\circ} 05^{\prime}\right.$ W). Coll and subm 1975 by C Gabet. Assoc with Artenac ceramics. Comment: confirms old age of Artenac culture.

Gif-3702. Chatelaillon E 7, Charente Maritime

$820 \pm 90$

Human bone found in monolithic sarcophagus of ancient necropolis of Chatelaillon $\left(46^{\circ} 4^{\prime} \mathrm{N}, 1^{\circ} 5^{\prime} \mathrm{W}\right)$. Coll and subm 1975 by C Gabet. Comment: expected date: 7 th century AD. Date may indicate re-use in Middle ages of this Merovingian sarcophagus.

\section{La Sauzaie series, Soubise, Charente Maritime}

Marine shells from Peu-Richardien site $\left(45^{\circ} 53^{\prime} \mathrm{N}, 0^{\circ} 50^{\prime} \mathrm{W}\right)$. Coll by J Gachima and subm 1972 by Y Guillien.

Gif-2608. La Sauzaie, 4

$$
4410 \pm 120
$$

Marine shells from basal level, beginning of settlement.

Gif-2610. La Sauzaie, 6

$4360 \pm 120$

Marine shells.

Gif-2609. La Sauzaie, 9

$$
2930 \pm 100
$$

Marine shells from ashy level. Comment: date suggests late occupation of site.

General Comment: dates agree with Gif-1557:4500 \pm 140 previously obtained for site. Gif-2610 (4360 \pm 120$)$ dates end of Peu-Richardien culture.

Gif-2414. La Grotte du Loup, Brive, Corrèze

$\geqslant 40,000$

Carbonaceous earth from Level $5 \mathrm{~b}$ in Grotte du Loup $\left(45^{\circ} 09^{\prime} \mathrm{N}, 1^{\circ}\right.$ $32^{\prime}$ E). Coll and subm 1972 by G Maziere, Limoges. Level attributed to Chatelperronian, ie, Upper Perigordian period.

\section{Grotte de Pégourié series, Caniac du Causse, Lot}

Samples from Grotte de Pégourié ( $\left.44^{\circ} 37^{\prime} 30^{\prime \prime} \mathrm{N}, 1^{\circ} 39^{\prime} \mathrm{E}\right)$. Coll and subm 1972-1973 by R Seronie-Vivien, Bordeaux.

Gif-2568. Grotte de Pégourié, C5

Gasteropod shells from Layer 5. Assoc with Azilian industry.

\section{Gif-2822. Grotte de Pégourié, C7 $12,250 \pm 350$}

Charcoal from Layer 7. From earliest Azilian occupation, just above late Magdalenian.

General Comment: wide range of dates between these two levels may be due to some recent contamination.

\section{Grotte des Cloups series, Caniac du Causse, Lot}

Charcoal from Grotte des Cloups (44 $\left.37^{\prime} 55^{\prime \prime} \mathrm{N}, 1^{\circ} 39^{\prime} 45^{\prime \prime} \mathrm{E}\right)$. Coll and subm 1975 by J Clottes, Dir Antiquités Prehist Midi-Pyrénées, Foix, Ariège. 
Gif-3569. Grotte des Cloups, Level 5

Charcoal from Late Bronze age level.

Gif-3568. Grotte des Cloups, Level 2

$3210 \pm 110$

Charcoal from Middle Bronze age level. Comment: consistent with dates of Middle Bronze age of Quercy region; Grotte du Noyer, $3150 \pm$ 110, Gif-1631, and $3250 \pm 110$, Gif-1159 (R, 1972, v 14, p 280-320).

Gif-3578. Grotte de la Cabillière, Saint-Simon, Lot $\quad 2840 \pm 100$

Charcoal from hearth in Late Bronze age level $\left(44^{\circ} 42^{\prime} 30^{\prime \prime} \mathrm{N}, 1^{\circ} 50^{\prime}\right.$ E). Coll by L Genot and subm 1975 by J Clottes.

Gif-2396. "Petite Caougne", Niaux, Ariège

$3110 \pm 100$

Charcoal from Chassean site $\left(42^{\circ} 51^{\prime} \mathrm{N}, 1^{\circ} 35^{\prime} \mathrm{E}\right)$ at foot of cliff. Coll and subm 1979 by J Clottes.

\section{Capdenac-Le-Haut series, Lot}

Charcoal from Neolithic site with Chassean level, $1 \mathrm{~m}$ thick, contain-

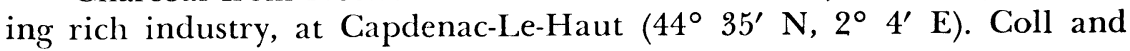
subm 1972-1975 by J Clottes.

Gif-2632. Capdenac-Le-Haut $5100 \pm 140$

Charcoal from upper part of Chassean level.

Gif-3713. Capdenac-Le-Haut, Level 9 $4870 \pm 110$

Charcoal.

Gif-3714. Capdenac-Le-Haut, Level 16 $5190 \pm 120$ Charcoal.

Gif-3715. Capdenac-Le-Haut, Level 20 $5140 \pm 120$

Charcoal.

General Comment: dates indicate very short occupation of site; impossible to establish chronography of this important Chassean layer.

Gif-3712. Capdenac-Le-Haut, Lot, Excavation B $\quad 2640 \pm 90$

Charcoal from 2nd excavation, $10 \mathrm{~m}$ from main excavation. Very rich Late Bronze Age III industry. Coll and subm 1975 by J Clottes.

\section{Grotte des Eglises series, Ussat, Ariège}

Charcoal from Magdalenian site $\left(42^{\circ} 49^{\prime} \mathrm{N}, 1^{\circ} 37^{\prime} \mathrm{E}\right)$. Coll and subm by J Clottes.

Gif-3922. Grotte des Eglises, Layer 1 $2160 \pm 90$

Charcoal. Comment: date conflicts with expected Bronze age.

Gif-4360. Grotte des Eglises, hearth $4610 \pm 120$

Charcoal from site without industry, separated from Layer 1 by sterile level. Comment: probable movement of Neolithic intrusion into supposed Bronze age level. 
Gif-3923. Grotte des Eglises, Layer 8 bis

$12,900 \pm 220$

Charcoal from Magdalenian level. Comment: date agrees with 1st age, $11,800 \pm 500$, Gif-1434, obtained for level, using undersized sample (R, 1972, v 14, p 287).

Grotte de Gazel series, Sallèles Cabardès, Aude

Grotte de Gazel $\left(43^{\circ} 19^{\prime} \mathrm{N}, 2^{\circ} 25^{\prime} \mathrm{E}\right)$ contains all archaeol levels from Medieval to Upper Magdalenian. Charcoal coll and subm 1970 by D Sacchi, CNRS, Carcassonne, Aude.

Gif-2401. Grotte de Gazel, Level 4

$6810 \pm 130$

Charcoal. Comment: attributed to Epipaleolithic but probably contaminated by overlying level.

Gif-2653. Grotte de Gazel, Level 5

$$
10,080 \pm 190
$$

Charcoal from important Epimagdalenian horizon.

Gif-2654. Grotte de Gazel, Level 6

$$
10,760 \pm 190
$$

Charcoal from important Epipaleolithic horizon.

Gif-2655. Grotte de Gazel, Level 7

$15,070 \pm 270$

Charcoal from basal level of stratigraphy. Corresponds to Magdalenian IV-V.

General Comment: 1st chronologic data for Upper Paleolithic in W Languedoc. Dates agree with archaeol records.

\section{Grotte de Canecaude series, Villardonnel, Aude}

Samples from Canecaude cave $\left(43^{\circ} 19^{\prime} \mathrm{N}, 2^{\circ} 19^{\prime} \mathrm{E}\right)$. Coll and subm 1969-1972 by D Sacchi.

Gif-2402. Canecaude, Level 2/2

$$
670 \pm 80
$$

Charcoal from hearth. Comment: date indicates intrusion of recent charcoal in this level attributed to Magdalenian III.

Gif-2708. Canecaude, Level 2/2

$$
14,230 \pm 160
$$

Bones from Magdalenian III level. Comment: dates agree with archaeol evidence.

Gif-2155. Canecaude, Level 2/1

$\mathbf{2 0 , 8 0 0} \pm \mathbf{5 5 0}$

Bones from Magdalenian Level 2/1. Comment: date in strong conflict with expected age; may be explained by mixing with lower levels due to difficulty of coll in one level only.

Gif-2709. Canecaude, Level 3

$22,230 \pm 330$

Bones from Upper Aurignacian level.

Gif-2710. Canecaude, Level 4

Bones from Aurignacian level.

$\mathbf{2 4 , 5 1 0} \pm 400$ 
Gif-2415. La Cauna d'Arques, Aude, A

$\mathbf{8 9 2 0} \pm \mathbf{2 0 0}$

Shells (Helix) from Level 2b, from "escargotière" in cave $\left(42^{\circ} 55^{\prime} \mathrm{N}\right.$, $2^{\circ} 23^{\prime} \mathrm{E}$ ). Coll and subm 1972 by D Sacchi. Comment: date agrees with assoc Mesolithic industry (Sacchi, 1972).

Gif-2416. La Cauna d'Arques, Aude, B

$$
1480 \pm 90
$$

Charcoal from crucible of bronze foundry contiguous to Mesolithic "escargotière", Level 2 (Gif-2415:8920 \pm 200 ). Coll and subm 1971 by D Sacchi. Comment: date agrees with assoc protohistoric ceramics.

Gif-2758. La Fontaine, Place du Palais, Avignon, Var $3750 \pm 110$

Charcoal in silt from open-air site at foot of Palais des Papes $\left(43^{\circ} 56^{\prime}\right.$ $\mathrm{N}, 4^{\circ} 48^{\prime} \mathrm{E}$ ). Coll and subm 1972 by J Courtin, Marseille. Comment: assoc with 20 to 30 entire vessels, with typical Chalcolithic characters.

Gif-2759. San Sebastien, Plan de la Tour, Var

$750 \pm 110$

Burned bones from Dolmen 2, Layer $1\left(43^{\circ} 21^{\prime} \mathrm{N}, 6^{\circ} 33^{\prime} \mathrm{E}\right)$. Coll and subm 1972 by J Courtin. Comment: dolmen attributed to Chalcolithic period. Date probably corresponds to late occupation.

\section{La Baume Fontbregoua series, Salernes, Var}

Charcoal from La Baume Fontbregoua $\left(43^{\circ} 33^{\prime} \mathrm{N}, 6^{\circ} 14^{\prime} \mathrm{E}\right)$ coll and subm 1972-1973 by J Courtin. Site is archaeol sequence, $8 \mathrm{~m}$ thick, from SE France, containing level of Neolithic artifacts, $4 \mathrm{~m}$ thick, with Chassean, Cardial, and Chalcolithic pottery levels, and level of Mesolithic and late Paleolithic hearths, $4 \mathrm{~m}$ thick, at bottom. Excavation begun in 1969 and continued in 1972-1973. Some dates obtained for Neolithic levels are pub (R, 1974, v 16, p 32).

Gif-2432. La Baume Fontbregoua, 9 $5100 \pm 110$

Charcoal from Late Chassean industry.

Gif-2433. La Baume Fontbregoua, 12 $4880 \pm 110$

Charcoal from Late Chassean industry.

Gif-2434. La Baume Fontbregoua, 18

$5040 \pm 110$

Charcoal from Late Chassean industry separated from lower levels by falling stones.

Gif-2435. La Baume Fontbregoua, 21 $5430 \pm 120$

Charcoal from layer with abundant Chassean ceramics.

Gif-2436. La Baume Fontbregoua, 25

$5600 \pm 120$

Charcoal from Chassean industry.

Gif-2437. La Baume Fontbregoua, 27

$5420 \pm 120$

Charcoal from Early Chassean industry, separated from lower level by falling stones.

Gif-2754. La Baume Fontbregoua, 31 $5660 \pm 130$

Charcoal from Middle Neolithic with Early Chassean industry. 
Gif-2755. La Baume Fontbregoua, 33

$5610 \pm 130$

Charcoal from Middle Neolithic with Early Chassean industry.

Gif-2756. La Baume Fontbregoua, 40

$5690 \pm 130$

Charcoal from Early Neolithic with late Cardial industry.

Gif-2757. La Baume Fontbregoua, 42

$5690 \pm 190$

Charcoal from Early Neolithic with late Cardial industry.

Gif-2988. La Baume Fontbregoua, 43

Charcoal assoc with late Cardial Neolithic industry.

Gif-2989. La Baume Fontbregoua, 45

Charcoal from Early Neolithic with Cardial industry.

Gif-2990. La Baume Fontbregoua, 47

$6700 \pm 100$

Charcoal from Early Neolithic with Cardial industry, separated from lower level by $1 \mathrm{~m}$ sterile sand.

Gif-2991. La Baume Fontbregoua, 51

$7600 \pm 100$

Charcoal from Mesolithic industry.

Gif-2992. La Baume Fontbregoua, 54

$8400 \pm 110$

Charcoal from Mesolithic industry.

Gif-2993. La Baume Fontbregoua, 61

$9570 \pm 120$

Charcoal from Early Mesolithic without microliths.

Gif-2753. La Baume Fontbregoua, Hearth A $\quad 9410 \pm 160$

Charcoal from $\mathrm{S}$ excavation. Early Mesolithic with archaic lithic industry, without domestic fauna or pottery.

Gif-2994. La Baume Fontbregoua, 70

$11,200 \pm 150$

Charcoal from Late Paleolithic, with bone industry.

General Comment: thickness of upper part of site provides good chronology of evolution of Neolithic industries in SE France. Dates agree with archaeol data for whole sequence (Courtin, 1973).

\section{Entzheim series, Sablière Oesch, Bas Rhin}

Charcoal from pits of open-air Neolithic site at sandpit Oesch, Entzheim $\left(48^{\circ} 32^{\prime} 40^{\prime \prime} \mathrm{N}, 7^{\circ} 39^{\prime} 45^{\prime \prime} \mathrm{E}\right)$. Coll by G Schmitt and subm 1971 by A G Thévenin, Dir Antiquités Prehist Alsace, Strasbourg (Thévenin, 1970).

Gif-2246. Entzheim, Pit 21

$4300 \pm 190$

Charcoal at depth 0.8 to $1 \mathrm{~m}$ in loam. Assoc with late Neolithic ceramics called Micheleberg. Inert $\mathrm{CO}_{2}$ added for measurement.

Gif-2247. Entzheim, Pit 2 I

$4340 \pm 190$

Charcoal. Inert $\mathrm{CO}_{2}$ added for measurement. 
Gif-2386. Entzheim, Pit 55

$\mathbf{3 8 5 0} \pm 110$

Charcoal assoc with ceramics of Roessen-Michelsberg limit. Comment: date expected to be a little older than Gif-2246 and -2247. Sample probably contaminated.

Gif-2248. Entzheim, Pit 40

$2390 \pm 110$

Charcoal at depth 0.8 to $1 \mathrm{~m}$ in loam. Comment (AGT): date explained by re-occupation of site during La Tène period.

\section{Oberlag series, Haut Rhin}

Charcoal from Rockshelter Mannlefelsen I., Oberlag $\left(47^{\circ} 27^{\prime} 25^{\prime \prime} \mathrm{N}\right.$, $7^{\circ} 14^{\prime} 21^{\prime \prime}$ E) (Thévenin and Sainty, 1972). Subm 1972 by A G Thévenin. Lowest level of cave was dated by Nancy lab:10,220 \pm 330 (unpub) and intermediate levels by Lyon lab (R, 1978, v 20, p 19-57).

Gif-2634. Oberlag, $O$ B 5

$5140 \pm 140$

Charcoal from Level $\mathrm{G}$ assoc with Rubane-Hinkelstein ceramics. Coll by J Sainty. Comment: date agrees with archaeol data for this industry.

Gif-2387. Oberlag, O B, Sq V5

$9030 \pm 160$

Charcoal from Level Q I assoc with Epipaleolithic Tardenoisian industry. Coll by A G Thévenin.

Gif-2530. Rochedane, Villars-sous-Dampjoux, Doubs $9210 \pm 120$

Bone from Level B in rockshelter at Rochedane $\left(47^{\circ} 42^{\prime} \mathrm{N}, 5^{\circ} 25^{\prime} \mathrm{E}\right)$. Azilian industry with engraved pebble (Thévenin and Sainty, 1972). Coll and subm 1972 by A G Thévenin. Comment: dates for lowest level given by Evin, Marien, and Pachiaudi, 1978.

\section{Grotte de Taforalt series}

\section{B. Morocco}

Grotte de Taforalt $\left(34^{\circ} 49^{\prime} \mathrm{N}, 2^{\circ} 24^{\prime} \mathrm{W}\right)$ is in mt group of BeniSnassen, alt $750 \mathrm{~m}, 60 \mathrm{~km}$ NW Oujda. Site has most extensive and complete stratigraphy of Upper, Middle Paleolithic, and Epipaleolithic of Maghreb. From upper levels, above Epipaleolithic-Aterian transition, dates were obtained on charcoal; from lower levels charcoal remains were very rare and gasteropod shells were dated. Samples coll and subm from 1970 to 1972 by J Roche, CNRS, Paris.

\section{Gif-2267. Grotte de Taforalt, Level 10,} F G 16-17

$13,140 \pm 150$

Charcoal. Early Epipaleolithic level.

Gif-2268. Grotte de Taforalt, Level 10, H 16-17 $\quad 13,500 \pm 150$ Charcoal.

Gif-2269. Grotte de Taforalt, Level 11, F G 16-17

$14,130 \pm 160$

Charcoal, two determinations.

$14,020 \pm 160$ 
Gif-2270. Grotte de Taforalt, Level 11, H 16-17 $\quad 15,240 \pm 180$

Charcoal.

Gif-2271. Grotte de Taforalt, transition Level

Charcoal.

Gif-2272. Grotte de Taforalt, Level 12, H 16-17 $\quad 15,460 \pm 180$

Charcoal.

Gif-2273. Grotte de Taforalt, Level 12,

$$
\text { I J K 18-19 } \quad 15,500 \pm 180
$$

Charcoal. Comment: date for same level obtained by Köln Lab: $16,050 \pm 190(\mathrm{Kn}-1559)$.

Gif-2586. Grotte de Taforalt, Level 14, H I $21,100 \pm 400$

Charcoal.

Gif-2587. Grotte de Taforalt, Levels 15-16,

$$
\text { G 13-14 }
$$$$
21,900 \pm 400
$$

Carbonaceous earth.

$$
\geqslant 32,370+2470
$$

Gif-2276. Grotte de Taforalt, H K $22 \quad \delta^{13} C=-11.8 \%$ o

Gasteropod shells, in transition level corresponding to EpipaleolithicAterian contact.

\section{Gif-2277. Grotte de Taforalt, Level 19, G K 19-20}

$$
\begin{array}{r}
\geqslant \mathbf{3 4 , 5 5 0}+\mathbf{3 2 0 0} \\
-\mathbf{2 2 8 0} \\
\delta^{13} C=-7.5 \% 0
\end{array}
$$

Gasteropod shells assoc with Upper Aterian industry.

Gif-2588. Grotte de Taforalt, base, Level 19, M 20-21 $\geqslant 40,000$ Carbonaceous earth.

Gif-2589. Grotte de Taforalt, top, Level 19, M 20-21 $\geqslant 40,000$ Carbonaceous earth.

Gif-2278. Grotte de Taforalt, Level 21, I K 19-21

$19,080 \pm 250$

$19,400 \pm 250$ $\delta^{13} C=-7.6 \%$ o

Gasteropod shells, two determinations.

Gif-2279. Grotte de Taforalt, Level 23, G K 20-21

$$
\begin{aligned}
& \geqslant 40,000 \\
& \delta^{13} C=-7.6 \% \text { o }
\end{aligned}
$$

Gasteropod shells assoc with Aterian industry.

Gif-2280. Grotte de Taforalt, Level 26, I K 19-2I

Gasteropod shells. 
General Comment: overlying levels previously dated by Sa-13: 10,800 \pm 400 Level 2, L-399 E: 11,900 \pm 240 Necropolis level, Sa-14: 12,070 \pm 400 Level 6, Sa-15: 10,500 \pm 400 Level 8 (R, 1964, v 6, p 233-250). Dates provide coherent chronology of occupation of cave by Epipaleolithic Mangrabins. Dates for shells are questionable. In Taforalt stratigraphy, two dates for Aterian Levels 21 and 26, respectively, 19,080 (Gif-2278) and 21,860 (Gif-2280) are obviously aberrant. Thinness of gasteropod shells prohibited complete removal of external part by acid pretreatment. Gif2276, 32,370 and Gif-2277, 34,550 BP are only min ages (Roche, 1976; Delibrias and Roche, 1976).

\section{Grotte des Contrebandiers series, Temara}

Site is on Atlantic coast, in marine terrace at alt $14 \mathrm{~m}, 17 \mathrm{~km} \mathrm{SW}$ Rabat $\left(33^{\circ} 24^{\prime} \mathrm{N}, 7^{\circ} \mathrm{W}\right)$. Grotto was filled with abundant remains of Epipaleolithic industry, and in Aterian level at bottom, inferior human maxilla and cranium, "The man of Temara", were found (Roche and Texier, 1976).

Gif-2576. Grotte des Contrebandiers, Level 8,

$$
\text { J K } 20
$$

Marine shells. U-Th date: $137,000 \pm 17,000 \mathrm{yr}$.

Gif-2577. Grotte des Contrebandiers, Level 8, $12,500 \pm 170$ J K 20

$\delta^{13} C=-24.7 \%$ 。

Bone splinters.

Gif-2578. Grotte des Contrebandiers, Level 9, 35,200 \pm 2100 J K 20

$\delta^{1 s} C=+0.4 \%$ o

Marine shells. U-Th date: $138,000 \pm 17,000 \mathrm{yr}$.

Gif-2579. Grotte des Contrebandiers, Level 9, J H 20

$14,460 \pm 200$

Bone splinters.

Gif-2580. Grotte des Contrebandiers, Level 10, $12,320 \pm 600$ J H $20 \quad \delta^{13} C=-30.8 \%$ o

Bone splinters. Undersized sample; inert $\mathrm{CO}_{2}$ added for measurement. Comment: probably not good date.

Gif-2581. Grotte des Contrebandiers, Level 11, G $20>40,000$ Marine shells.

Gif-2582. Grotte des Contrebandiers, Level 11, J 20

$24,500 \pm 600$

Bone splinters.

Gir-2583. Grotte des Contrebandiers, Level 12, G 20

$12,170 \pm 160$

Bone splinters. 
Gif-2584. Grotte des Contrebandiers, Level 12, G $20>35,000$

Marine shells.

\section{Gif-2585. Grotte des Contrebandiers, Level 12, E 18}

$23,700 \pm 1000$

Carbonaceous earth.

General Comment: sequence is dated by ages of bones which agree with archaeol research. Gif-2582: 24,500 BP for bones is confirmed by Gif-2585: 23,700 BP obtained for carbonaceous material and validates results. Yet, Gif-2583: 12,170 вр is aberrant for Level 12 and may be explained in two ways: either samples Gif-2582 and -2583 were confused or bones intruded from Level 10 into Level $12 .{ }^{14} \mathrm{C}$ dates for shells are unacceptable as they disagree with dates of bones and U-Th dates for same shells. Dated shellfish, then, were not consumed by inhabitants of cave but come from walls of cave, deposited into different levels during occupation of site. Gif-2576: 22,630 BP demonstrates recent contamination of shells in upper Level 8. Moreover, marine terrace where cave is, dated at 137,000 yr old, which means it belongs to Ouljian transgression; U-Th dates are from Hoang, CFR, CNRS, Gif-sur-Yvette.

\section{Tarfaya region series}

Littoral Neolithic sites are frequently found in SW Morocco. Region near Tarfaya, E Juby Cape was surveyed and many samples were coll in 1973-1974 by N Petit-Maire, CNRS, Marseille Luminy and L Ortlieb, ORSTOM, Paris (Delibrias, Ortlieb, and Petit-Maire, 1976).

Gif-2905. Tarfaya region, Site $F, 4$ Burned shell (Cymbium sp) from beach $\left(27^{\circ} 58^{\prime} \mathrm{N}, 12^{\circ} 49^{\prime} \mathrm{W}\right)$.

Gif-2906. Tarfaya region, Site F, 4

Human bones.

Gif-2908. Tarfaya region, Site 11/2

Charcoal at depth $0.7 \mathrm{~m}$ in dune on beach $\left(28^{\circ} 01^{\prime} \mathrm{N}, 12^{\circ} 30^{\prime} \mathrm{W}\right)$.

Modern

Gif-2909. Tarfaya region, Site $11 / 1$ a

$10,430 \pm 180$

Human bones in burial site, depth $1.3 \mathrm{~m}$.

Gif-2910. Tarfaya region, Site 11/1b

$6100 \pm 120$

Charcoal at depth $0.1 \mathrm{~m}$. Comment: Gif-2910 and -2909 are superimposed in dune; Gif-2908 is dispersed more superficially. If date of bones is accurate and dates for collagen of bones are generally reliable, especially in arid regions, charcoal from ancient superficial site was probably buried at time of interment $6000 \mathrm{yr}$ ago. Date of charcoal suggests that human occupation at Juby Cape dates to 10,000 yr ago (Charon, Ortlieb, and Petit-Maire, 1973).

Gif-2911. Tarfaya region, Site 11

$4450 \pm 110$ bones.

Charcoal on beach $\left(27^{\circ} 45^{\prime} \mathrm{N}, 13^{\circ} 43^{\prime} \mathrm{W}\right)$ assoc with hippopotamus 
Gif-2821. Tarfaya region

$3300 \pm 100$

Charcoal in great littoral Kjokenmödding (shell midden), $1 \mathrm{~km} \mathrm{~S}$ Tafaya. Coll by M Mateu.

Gif-2912. Foum-el-Arjam

$2360 \pm 250$

Human bones in barrow at Foum-el-Arjam, S Zagora $\left(30^{\circ} 22^{\prime} \mathrm{N}, 5^{\circ}\right.$ $\left\ulcorner 0^{\prime}\right.$ W). Coll 1971 by A Simoneau. Subm 1973 by N Petit-Maire.

\section{Gif-3013. El Ouaâr, Site G}

$3550 \pm 120$

Shells (Mytilus sp) at depth $1 \mathrm{~m}$, coll from Neolithic site at El Ouaâr $\left(28^{\circ} 10^{\prime} \mathrm{N}, 11^{\circ} 52^{\prime} \mathrm{W}\right)$.

Mauritania

\section{West Africa}

Important Neolithic sites with numerous sepultures and rich industry, similar to those in SW Morocco, were found distributed along ancient shores of Nouakchottian Sea in Mauritania. Two main sites, Chami and Tintane, were studied from 1970-1972 by N Petit-Maire (human morphol, ecol, and vertebrate paleontol), H Faure and L Hebrard, CNRS, Marseille (geol), P Elouard, Fac Sci, Lyon (vertebrate paleontol), E Maquet and C Lecour (ceramics). Because of wind erosion many skeletons were partly uncovered and splinters of bone were found scattered on ground surface. Dated bones came mainly from skeletons still underground at depth 10 to $100 \mathrm{~cm}$. Some Kjökkenmödding were also dated. In order to make statistical study of human ecology in Late Quaternary of W Africa, many dates were necessary to demonstrate that samples were contemporaneous (Petit-Maire, 1979).

\section{Chami series}

Samples coll from Neolithic sites on fossil dunes, $30 \mathrm{~km}$ around Chami well $\left(20^{\circ} 4^{\prime} \mathrm{N}, 15^{\circ} 58^{\prime} \mathrm{W}\right)$. Except where otherwise stated, samples coll 1970-1971 by N Petit-Maire.

Gif-1762. Chami, Fossil Dune 15a

$3100 \pm 120$

Faunal bones.

Gif-1856. Chami, Fossil Dune 15a $3950 \pm 80$

Shells.

Gif-1970. Chami, Fossil Dune 15a $\quad 3650 \pm 140$

Organic remains in ceramic debris introduced into clay at time of manufacture.

Gif-2161. Chami, Fossil Dune 15a, H $7 \quad 2170 \pm 100$

Human bones.

Gif-2162. Chami, Fossil Dune 15a, H $8 \quad 2500 \pm 100$

Human bones.

Gif-2166. Chami, Fossil Dune 15a, G $2290 \pm 130$

Bones from large mammal. 
Gif-2488. Chami, Fossil Dune 15a

$3450 \pm 110$

Shell (Cymbium) assoc with skull and jar.

Gif-2492. Chami, Fossil Dune 15a

$3500 \pm 120$

Charcoal remains in sand (? hearth) assoc with gray ceramics. Coll 1971 by G Delibrias.

Gif-2163. Chami, Fossil Dune 15b, $T_{1}-1$

$2100 \pm 180$

Human bones.

Gif-2164. Chami, Fossil Dune 15b, $T_{1}-2 \quad 2360 \pm 100$

Shell (Cymbium) lying on ground above Sepulture $\mathrm{T}_{1}$. Comment: large Cymbium shells were often used for tomb marking. Dates obtained for shell and bones (Gif-2163) agree well.

Gif-2489. Chami, Fossil Dune 15b

$2090 \pm 120$

Human bones.

Gif-2168. Chami, Fossil Dune 6, $T_{3}$ $\delta^{13} C=-16.8 \%$ o

Human bones.

Gif-2167. Chami, Fossil Dune 6, top

$2450 \pm 130$

Human bones from sepulture at top of mound.

$3310 \pm 240$

Gif-1764. Chami, Fossil Dune 7, 5

$\delta^{13} C=-23.1 \%$

Faunal bones found on surface. Comment: unrelated to ancient site occupation.

Gif-1765. Chami, Fossil Dune 7, 6

$2500 \pm 100$

Human bones dispersed on surface.

$\delta^{13} C=-12.6 \%$

Gif-2165. Chami, Fossil Dune 7

$6580 \pm 350$

Human bones from top of dune. Comment: inactive $\mathrm{CO}_{2}$ was added for measurement. One of the earliest dates found for Mauritania; requires confirmation.

Gif-3060. Chami, Fossil Dune 7, top

$3530 \pm 220$

Human bones.

Gif-2333. Chami, Fossil Dune 7, GP 11

$4190 \pm 130$

Human bones dispersed on surface.

Gif-2334. Chami, Fossil Dune 7

$1870 \pm 240$

Organic remains in ceramic debris. Comment: undersized sample, inactive $\mathrm{CO}_{2}$ was added for measurement.

Gif-2486. Chami, Fossil Dune 8

$3850 \pm 120$

Human bones from sepulture with two skeletons.

$\delta^{13} C=-16.5 \%$ 
Gif-2487. Chami, Fossil Dune 8

$3220 \pm 110$

Shell (Cymbium) beneath skull.

Gif-2491. Chami, Fossil Dune 11a

$2960 \pm 110$

Ostrich eggshell assoc with pearl in Sepulture T8.

Gif-1763. Chami, Fossil Dune 1

$2450 \pm 110$

Human bones.

Gif-1857. Chami, Fossil Dune 1

$2380 \pm 200$

Human bones. Comment: inactive $\mathrm{CO}_{2}$ was added for measurement.

Gif-1859. Chami, Fossil Dune 1

$1520 \pm 140$

Human bones.

\section{Tintane series}

Tintane site is similar to Chami site because of abundant Neolithic industry. At Tintane, sepultures appear with structured tombs and vertebrate faunal remains are absent. Samples coll 1970-1971 by N Petit-Maire except where otherwise stated.

Gif-1761. Tintane, Txx, 2

$2470 \pm 100$

$\delta^{13} C=-13.0 \%$

Human bones from Sepulture Txx, at Tintane necropolis $\left(20^{\circ} 55^{\prime} \mathrm{N}\right.$, $\left.16^{\circ} 40^{\prime} \mathrm{W}\right)$.

Gif-1824. Tintane, Txx, 52

$2460 \pm 100$

Organic remains in ceramic debris, around Sepulture Txx. Comment: date agrees well with bones from Gif-1761.

Gif-2484. Tintane, $\mathbf{S}_{1}$

$3240 \pm 100$

Human bones from Skeleton $S_{1}$. Coll 1971 by J M Casiez, Fac Sci, Paris.

Gif-2490. Near Tintane

Modern

Ostrich egg shell from surface, near Tintane $\left(20^{\circ} 49^{\prime} \mathrm{N}, 16^{\circ} 07^{\prime} \mathrm{W}\right)$, near three large, half-buried, unbroken clay jars. Coll 1971 by $G$ Delibrias. Comment: ostriches disappeared very recently from this region; egg shells are unrelated to site.

Gif-2493. Near Tintane

$$
\begin{array}{r}
2810 \pm \mathbf{1 0 0} \\
\delta^{13} C=-21.0 \%
\end{array}
$$

Fine charcoal remains in sand at depth ca $20 \mathrm{~cm}$, same site as Gif2490, near three large unbroken clay jars. Coll 1971 by G Delibrias.

Gif-2485. Tintane, N Fossil dune

$3930 \pm 80$

Oyster shell on top of N Fossil dune.

Gif-2494. Baie de L'Etoile

$5730 \pm 120$

Shell (Arca senilis) from Kjökkenmödding, $15 \mathrm{~km} \mathrm{~N}$ Nouadhibou, $\left(21^{\circ} 02^{\prime} \mathrm{N}, 17^{\circ} 02^{\prime} \mathrm{W}\right)$, ca $3 \mathrm{~m}$ above msl. Coll 1971 by G Delibrias. Comment: dates occupation of Nouakchottian shoreline. 


\section{Baie de Saint Jean series}

Kjökkenmödding with shells (mainly Arca senilis) and some ceramics but no furniture, chipped flint implements, or sepultures, at Cape Tafarit. Coll and subm 1971 by G Delibrias.

\section{Gif-2496. Tafarit 1}

$$
3220 \pm 110
$$

Shell (Arca senilis) from surface. Comment: Kjökkenmödding nearby was dated at 6130 вP, Ly-345. Large discrepancy between these dates may show successive occupations.

\section{Gif-2524. Tafarit 2}

$$
3410 \pm 110
$$

Shell (Arca senilis) at depth $1.5 \mathrm{~m}$ beneath Gif-2496. Comment: shells mixed with black sand represent lowest level of midden deposit on sandbar.

\section{Gif-2497. Tafarit 3}

$$
3080 \pm 110
$$

Sandy black soil from basal level of Kjökkenmödding dated by Gif2496 and -2524. Gif-2524 assoc with this sample.

General Comment: dates indicate 400-yr occupation of site. Younger date for soil is probably age of last surface soil that gradually penetrated through shells, because lst $\mathrm{m}$ is shell deposit only.

\section{Anate series, Agneitir Hills}

Sand dune bar, $10 \mathrm{~km}$ along ancient Nouakchottian gulf at 15 to $20 \mathrm{~m}$ above msl, covered by shells eaten by people living on coast at Anate, $24 \mathrm{~km}$ from Nouanghar, near Cap Timiris.

\section{Gif-2498. Anate}

$$
4350 \pm 120
$$

Shells (Arca senilis) from surface.

\section{Gif-2499. Anate}

$$
4670 \pm 130
$$

Shells (Arca senilis) at depth $1 \mathrm{~m}$.

General Comment: occupation lasted $600 \mathrm{yr}$ at most. $40 \%$ of shells removed by acid before dating.

\section{Atlantic Sahara}

Human occupation during Holocene between Seguiet el Hamra and Cape Blanc along Atlantic coast of Sahara is mostly characterized by numerous shell middens. Traces of occupation by man are also abundant along coastal sebkhas (dry lagoons). As noted in Mauritania, evolution of Saharan coast and of human occupation are often parallel and closely related to climatic variation. Samples coll $1973-1974$ by N Petit-Maire and L Ortlieb.

Gif-3259. Sebkha Amtal, 64280

$$
2000 \pm 90
$$

Shell (Arca senilis) in midden on banks of Sebkha Amtal $\left(23^{\circ} 54^{\prime} \mathrm{N}\right.$, $\left.16^{\circ} 13^{\prime} \mathrm{W}\right)$.

Gif-3260. Sebkha Akhful, RNT 114

$4300 \pm 110$ W).

\footnotetext{
Lagoonal shells in littoral sebkha, at depth $0.2 \mathrm{~m}\left(26^{\circ} 15^{\prime} \mathrm{N}, 14^{\circ} 25^{\prime}\right.$
} 

W).

Shell (Arca senilis) in littoral sebkha, on surface $\left(26^{\circ} 15^{\prime} \mathrm{N}, 14^{\circ} 26^{\prime}\right.$

Gif-3262. Medano Santiago, RNT 83

$3150 \pm 100$

Consumed shells (Mytilus sp) in continental deposit on coast, at depth $0.5 \mathrm{~m}\left(26^{\circ} 55^{\prime} \mathrm{N}, 13^{\circ} 30^{\prime} \mathrm{W}\right)$.

Gif-3264. Sebkha Amtal, RNT 138

$3070 \pm 100$

Marine shells at base of fossiliferous sandy deposit, at depth $0.3 \mathrm{~m}$ $\left(23^{\circ} 10^{\prime} \mathrm{N}, 16^{\circ} 10^{\prime} \mathrm{W}\right)$.

Gif-3265. Sebkha Amtal, RNT 137

$3300 \pm 100$

Marine shells in same deposit as Gif-3264, at depth of 0.1 to $0.3 \mathrm{~m}$.

Gif-3266. Sebkha Amtal, RNT 149

$3070 \pm 100$ $\left.10^{\prime} \mathrm{W}\right)$.

Littoral marine shells in green clay level, on surface $\left(23^{\circ} 10^{\prime} \mathrm{N}, 16^{\circ}\right.$

Gif-3466. Sebkha Amtal, H 1

$$
1780 \pm 100
$$

Human bones from sepulture in shell midden $\left(23^{\circ} 10^{\prime} \mathrm{N}, 16^{\circ} 10^{\prime} \mathrm{W}\right)$. Comment: date too young because of Neolithic cultural context.

Gif-3267. Sidi Bou-Maleh, RNT 200

$\geqslant 35,000$

Marine shells in sandstone on shore at high tide level $\left(28^{\circ} 24^{\prime} \mathrm{N}\right.$, $\left.11^{\circ} 24^{\prime} \mathrm{W}\right)$. Comment: corresponds to ancient high sea level, probably Ouljian transgression.

Gif-3269. Sebkha Laasailia, 63293

$2740 \pm 110$ $\left.30^{\prime} \mathrm{W}\right)$.

Human bones from sepulture under large flat stones $\left(26^{\circ} 55^{\prime} \mathrm{N}, 13^{\circ}\right.$

Gif-3464. Sebkha Laasailia, H 6

Modern

Human bones from sepulture in eolian sand above sebkha.

Gif-3465. Sebkha Laasailia, H 10

$3100 \pm 110$ sebkha.

Human bones from sepulture under large flat stones, on bank of

Gif-3461. Sebkha Edjaila, H 2

$3020 \pm 100$

Human bones from sepulture in dune sand $\left(21^{\circ} 45^{\prime} \mathrm{N}, 16^{\circ} 25^{\prime} \mathrm{W}\right)$.

Gif-3462. Sebkha Edjaila, H 3

$3310 \pm 110$

Human bones from sepulture near Gif-3463.

Gif-3514. Sebkha Edjaila, SE 166

$4440 \pm 110$

Lagoonal-marine shells at depth 0.2 to $0.5 \mathrm{~m}$, in bottom of sebkha, alt ca $-0.5 \mathrm{~m}\left(21^{\circ} 45^{\prime} \mathrm{N}, 16^{\circ} 25^{\prime} \mathrm{W}\right)$.

Gif-3510. Sebkha Edjaila, SE 161

$4850 \pm 110$

Shells (Cardium edule) interbedded in green clay at depth 0.15 to $0.5 \mathrm{~m}$, in bottom of sebkha, alt ca $\operatorname{lm}\left(21^{\circ} 45^{\prime} \mathrm{N}, 16^{\circ} 25^{\prime} \mathrm{W}\right)$. 
Gif-3508. Sebkha Edjaila, SE 170

$540 \pm 80$

Shells (Arca noae) from emerged lagoonal marine deposit at depth 0.3 to $0.5 \mathrm{~m}$, in bottom of sebkha $\left(21^{\circ} 48^{\prime} \mathrm{N}, 16^{\circ} 27^{\prime} \mathrm{W}\right)$, alt ca $0 \mathrm{~m}$. Comment: dates closure of the Nouackchottian paleogulf.

\section{Gif-3467. Sebkha Mahariat, H 11}

$1080 \pm 100$

Human bones from sepulture in shell midden $\left(22^{\circ} 16^{\prime} \mathrm{N}, 16^{\circ} 33^{\prime} \mathrm{W}\right)$. Comment: skull morphology very primitive; contradicts date.

Gif-3503. Sebkha Mahariat, 4741

$5360 \pm 120$

Consumed shells (Arca senilis) in fireplace on top of dune, on surface of offshore bar.

Gif-3504. Sebkha Mahariat, 4841

$6180 \pm 130$

Consumed shells (Arca senilis, Cymbium sp) in shell midden from surface at top of ancient dune.

Gif-3511. Sebkha Mahariat, SE 115

$5040 \pm 110$

Shells (Cardium edule) in sand, alt ca $1 \mathrm{~m}$, from bottom of sebkha, at depth $0.5 \mathrm{~m}\left(22^{\circ} 14^{\prime} \mathrm{N}, 16^{\circ} 35^{\prime} \mathrm{W}\right)$.

Gif-3512. Sebkha Mahariat, SE 149

$5250 \pm 110$

Shells from bottom of sebkha, close to surface, alt ca $2 \mathrm{~m}$.

\section{Gif-3515. Sebkha Mahariat, SE 108}

$4100 \pm 110$

Shells (Arca senilis) in salty clay sediment from surface of bottom of sebkha, alt ca $0 \mathrm{~m}$. Comment: dates dessication of lagoon.

Gif-3507. Sebkha Lemheiris, SE 138

$4350 \pm 150$

Shells (Cardium edule, Arca senilis) from surface of bottom of sebkha $\left(22^{\circ} 06^{\prime} \mathrm{N}, 16^{\circ} 48^{\prime} \mathrm{W}\right)$. Comment: dates dessication of lagoon.

\section{Gif-3505. Sebkha Lemheiris, SE 131}

$5810 \pm 120$

Lagoonal marine shells from green silty sand at depth $0.75 \mathrm{~m}$, in bottom of sebkha.

Gif-3509. Sebkha Lemheiris, SE 137

$6150 \pm 130$

Shells in littoral sandy deposit at depth $0.7 \mathrm{~m}$, in bottom of sebkha.

Gif-3463. Sebkha Lemheiris

$3740 \pm 130$

Human bones from sepulture under large flat stones $\left(22^{\circ} 06^{\prime} \mathrm{N}, 16^{\circ}\right.$ $48^{\prime} \mathrm{W}$ ) in dune sand.

Gif-34.68. Aadeim, $\mathbf{H} 4$

Modern

Human bones from sepulture near Aadeim ( $\left.25^{\circ} 48^{\prime} \mathrm{N}, 14^{\circ} 56^{\prime} \mathrm{W}\right)$.

Gif-3513. Aadeim, SE 73

$\geqslant \mathbf{3 5 , 0 0 0}$

Fragments of shells (Mytilus sp) in littoral silty deposit, $2 \mathrm{~m}$ above msl on beach of Aadeim. Comment: probably corresponds to Ouljian transgression. 


\section{Cameroon}

Gif-2232. Maroua

Charcoal from cutting-chipping factory cropping out of surface of Tsanaga plain at Maroua, Diamare Dept $\left(10^{\circ} 35^{\prime} \mathrm{N}, 14^{\circ} 15^{\prime} 14^{\prime \prime} \mathrm{E}\right)$. Coll and subm 1971 by A Marliac, ORSTOM, Yaoundé, Cameroon. Comment: late Neolithic industry.

\section{Aswad series}

D. Syria

Charcoal from preceramic Neolithic site, Tell Aswad $\left(33^{\circ} 35^{\prime} \mathrm{N}, 36^{\circ}\right.$ 32' E). Two phases, Aswad II and Aswad I are discernible from their flints. Industry of upper level is similar to that of Ramadi, Syria, and flint industry of lower level is similar to that of Munhata and Beidha sites, Palestine, assoc with baked-clay figurines of sitting women (de Contenson, 1976). Coll 1971-1972 by F Valla and subm by $\mathrm{H}$ de Contenson, Mission archéol Française Ras Shamra, Syria.

Gif-2373. Aswad, ẉ 1

$8560 \pm 110$ Aswad II.

Charcoal from last preserved level, in $\mathrm{W}$ part of site, at depth $0.3 \mathrm{~m}$,

Gif-2369. Aswad, E 1

$8540 \pm 110$

Charcoal from last occupation at depth $0.25 \mathrm{~m}$, in $\mathrm{E}$ part of site, Aswad II.

Gif-2370. Aswad, E 2

$9340 \pm 120$

Charcoal from middle of lower level, at depth $1.75 \mathrm{~m}$, Aswad I.

Gif-2371. Aswad, E 3

$9270 \pm 120$

Charcoal from lowest part of lower level, at depth $2.35 \mathrm{~m}$, Aswad I.

Gif-2372. Aswad, E 4

$9640 \pm 120$

Charcoal from beginning of occupation, at depth $2.45 \mathrm{~m}$, Aswad I.

Gif-2633. Aswad, E 5

$9730 \pm 120$

Charcoal from beginning of occupation, at depth $3.2 \mathrm{~m}$.

General Comment: W part of site was studied by Groningen lab. Dates are from $8650 \mathrm{BP}$ at $0.4 \mathrm{~m}$ depth, $\mathrm{GrN}-6676$, (compared with 8560 at $0.3 \mathrm{~m}$ depth, Gif-2373), to 8875 вр at $1.3 \mathrm{~m}$ depth, GrN-6678. Dates suggest shift of settlement $\mathrm{W}$-ward, older settlement in $\mathrm{E}$ part of site (de Contenson, 1973).

\section{Ghoraifé series}

Tell $15 \mathrm{~km} \mathrm{~N}$ of Tell Aswad. Three stages were recorded at this site in 1974: uppermost stage, frequently disturbed by more recent sepultures, and two lower Neolithic levels with lithic industry and clay objects. These two levels provide interesting correlations with Aswad and Ramad nearby. Charcoal coll and subm 1974 by $\mathrm{H}$ de Contenson. 
Gif-3371. Ghoraifé, GH 1

$6940 \pm 190$

Charcoal from depth $1.7 \mathrm{~m}$, dating end of continuous occupation of site. Comment: abundant rootlets suggest contamination.

Gif-3372. Ghoraifé, GH 2

$8150 \pm 190$

Charcoal from depth $3 \mathrm{~m}$. Phase II, faunal remains and bone industry, abundant obsidian.

Gif-3374. Ghoraifé, GH 4

$8400 \pm 190$

Charcoal from depth $5.5 \mathrm{~m}$. Beginning of ancient Phase I.

Gif-3375. Ghoraifé, GH 6

$8460 \pm 190$

Charcoal from depth $6 \mathrm{~m}$. Beginning of ancient Phase I.

\section{Gif-3376. Ghoraifé, GH 7}

$8710 \pm 190$

Charcoal from depth $6.2 \mathrm{~m}$. Phase I: bone industry and fauna scarce; lithic industry and baked clay figures similar to those of Aswad abundant in lower part of site.

General Comment: dates, together with those of Aswad and Ramad (GrN dates) establish continuous chronologic sequence from beginning of 8th millennium to middle of 6th millennium (de Contenson, 1976).

\section{Harrar series}

$$
\text { E. Ethiopia }
$$

Charcoal from megalithic monuments near Dobba $\left(4^{\circ} 32^{\prime} \mathrm{N}, 47^{\circ} 32^{\prime}\right.$ E). Coll and subm 1970-1973 by R Joussaume, Inst Paleontol Humaine, Paris.

Gif-1895. Tehaffe

$500 \pm 80$

Charcoal from Type II circular barrow, with circular rooms and exit passage. Comment: agrees with assoc industry.

Gif-2397. Tchalalaka

Modern

Charcoal from Type I monument, with rectangular room under large slab. Comment: Type I megalithic monument was expected to be older than Type II. Some are still used as places of worship (Joussaume, 1971).

\section{F. Central America}

\section{La Lagunita series, Guatemala}

Samples from Pre-Classic site of La Lagunita $\left(15^{\circ} 17^{\prime} \mathrm{N}, 91^{\circ} 50^{\prime} \mathrm{W}\right)$, in El Quiché area, W part of Guatemala Highlands. Coll and subm 19731978 by H Lehmann and A Ichon, CNRS, Mission Sci Franco-Guatemaltique, Guatemala (Ichon, 1977).

Gif-2834. La Lagunita $T_{2}, \mathbf{G 9},-110$

$3960 \pm 130$

Charcoal under stone flags, Level -110 , ceremonial Pyramid 5. Comment: no archaeol assoc. Date corresponds to Late Archaic period. 
Gif-2835. La Lagunita $T_{3,} S_{3}, \mathbf{- 5 9 0}$

$1930 \pm 100$

Charcoal with offerings from Level -150 , Pyramid 5. Comment: assoc with Pre-Classic industry. Corresponds to building stage of this structure.

Gif-4553. La Lagunita, Cave C 48, B8/25 $1610 \pm 80$

Charcoal in jar, $\mathrm{N}$ wall of underground room for offerings.

Gif-4554. La Lagunita, Cave C 48, E4/10 $1640 \pm 80$

Charcoal in jar, $\mathrm{W}$ wall of cave.

Gif-4555. La Lagunita, Cave C 48, C2/18 $1650 \pm 80$

Charcoal in jar, $S$ wall of cave.

Gif-4227. La Lagunita, Cave C 48/108 $1350 \pm 90$

Charcoal in earth floor of cave. Comment: disagrees with Late Classic ceramics from cave, well-dated by Gif- 4553 to -4555 .

Gif-4556. La Lagunita, EJ 16

$1900 \pm 90$

Charcoal in sterile level, from S-N trench in center of ceremonial place of La Lagunita site, at bottom of offering of stone statuettes.

Gif-4228. La Lagunita, C44/183

$1630 \pm 90$ -30 .

Charcoal in ceramic bowl, at level of Tomb 4 of "La Pila 3", Level

Gif-4229. La Lagunita, C44/B4-1

$1480 \pm 90$

Charcoal in another ceramic bowl, in Tomb 4, Level -260.

Gif-4230. La Lagunita, C47/513

$1640 \pm 90$

Charcoal in ceramic bowl with cover, in Pyramid 7, Level +520 .

Gif-4231. La Lagunita, C47/52

$1870 \pm 100$

Charcoal from hearth in Pyramid 7, Level +160 , assoc with PreClassic sepulture.

General Comment: results agree with archaeol date for Cave C 48, but Gif4227 and those from Pyramid 7 indicate that ceremonial center of $\mathrm{La}$ Lagunita was at its height during Classic period, ca 1600 to 1700 вP. Gif4556: $1900 \pm 90$ and Gif-4231: $1830+100$ confirm Pre-Classic settlement of site.

\section{San Andrès Sajcabaja series, Guatemala}

Samples from Pre-Classic monticule (artificial mound) $\left(15^{\circ} 10^{\prime} \mathrm{N}, 90^{\circ}\right.$ $57^{\prime} \mathrm{W}$ ) in Quiché area, W part of Guatemala Highlands. Coll and subm 1971 by A Ichon.

Gif-2833. San Andrès F2 (A2) $2580 \pm 100$

Charcoal in pit-hole, from oldest phase of building.

Gif-2832. San Andrès F2 (E3)

$2510 \pm 100$

Charcoal on top of stone box, probably used as sarcophagus. 
Gif-2478. San Andrès, Str 1

$1750 \pm 150$

Charcoal, from 4th phase of building of monticule.

General Comment: 3 of 5 building phases recognized in this structure were dated. Occupation of site lasted from Middle Pre-Classic to Late Pre-Classic periods. Dates agree well with bichrome ceramics industry found in these levels.

\section{Chimistan series, Guatemala}

Samples from Monticule 2 of site $\left(15^{\circ} 19^{\prime} \mathrm{N}, 91^{\circ} 41^{\prime} \mathrm{W}\right)$. Coll and subm 1971 by A Ichon.

Gif-2836. Chimistan $F_{3}$ G (-250) $\quad 2170 \pm 100$

Charcoal from level with abundant kitchen refuse just above sterile sand. Corresponds to first occupation of site.

Gif-2837. Chimistan $F_{3} J$ (-130)

$2200 \pm 100$

Charcoal from same level as Gif-2835.

General Comment: this monticule is dated from beginning of Late PreClassic period, as expected from ceramics.

Gif-2346. Nata, Coclé, Panama

$1640 \pm 90$

Charcoal from refuse level assoc with potsherds of Giron and Escota styles, near Nata $\left(8^{\circ} 8^{\prime} \mathrm{N}, 80^{\circ} 38^{\prime} \mathrm{W}\right)$. Coll and subm 1971 by R C Cooke, Inst Archaeol, London. Comment: this layer is overlain by level corresponding to beginning of polychrome pottery similar to those from Tonosi, Guatemala, suggesting close relations between countries.

\section{G. South America}

\section{Huancavelica series, Peru}

Charcoal from high alt sites in Huancavelica Dept, Central Andes. Coll 1970 and subm 1971 by M Julien and D Lavallée, CNRS, Paris. Dated to determine duration of "Intermediate Recent" period in Central Andes.

Gif-1987. Chuntamarka, 3110 B

$750 \pm 90$

Charcoal from floor of House B, $0.25 \mathrm{~m}$ below ground level $\left(12^{\circ} 38^{\prime}\right.$ $\mathrm{S}, 75^{\circ} 10^{\prime} \mathrm{W}$ ), alt $4400 \mathrm{~m}$.

Gif-1988. Laiwe, 3476

Charcoal from floor of House B, $0.4 \mathrm{~m}$ below ground level $\left(12^{\circ} 25^{\prime} \mathrm{S}\right.$, $75^{\circ} 9^{\prime} \mathrm{W}$ ), alt $3700 \mathrm{~m}$. Human burial place in same level.

Gif-2604. Laiwe, 3475 1988.

Charcoal from floor of House E, $0.4 \mathrm{~m}$ below ground level, near Gif-

Gif-2603. Shanki

$970 \pm 90$

Charcoal from hearth, $0.5 \mathrm{~m}$ below ground level $\left(12^{\circ} 25^{\prime} \mathrm{S}, 75^{\circ} 8^{\prime} \mathrm{W}\right)$, alt $3750 \mathrm{~m}$. 
Gif-1989. Cuto-Cuto, 3496 A

$780 \pm 90$

Charcoal, from Hearth A $\left(12^{\circ} 32^{\prime} \mathrm{S}, 75^{\circ} 10^{\prime} \mathrm{W}\right)$, alt $4000 \mathrm{~m}$.

Gif-2389. Cuto-Cuto, 3490 D

$750 \pm 90$

Charcoal, from House D.

General Comment: samples date period of "Intermediate Recent" in Central Andes between AD 1100/1200 and 1530 .

\section{Oloma Bajada series, Argentina}

Charcoal in artificial refuse mound with ceramics and bones, Prov Santiago del Estero (28 $\left.30^{\prime} \mathrm{S}, 63^{\circ} \mathrm{W}\right)$. Coll 1971 and subm 1972 by A M Lorandi. Pottery belongs to Sunchituyo culture that is similar to pottery of Quimili Paso, and to Averias culture.

Gif-2619. Oloma Bajada, Site I, 13 $340 \pm 90$

Charcoal from depth 0.4 to $0.6 \mathrm{~m}$.

Gif-2620. Oloma Bajada, Site II, 12 $460 \pm 90$

Charcoal from depth 0.2 to $0.4 \mathrm{~m}$.

Gif-2621. Oloma Bajada, Site II, 13 $\mathbf{5 3 0} \pm \mathbf{9 0}$

Charcoal from depth 0.4 to $0.6 \mathrm{~m}$.

Gif-2728. Lauta, Navarino Island, Chile

$280 \pm 90$

Shells from lower layer in one of numerous shell middens, Seno del Lauta, near Puerto Williams, Beagle Channel (54 $55^{\prime} \mathrm{S}, 67^{\circ} 40^{\prime} \mathrm{W}$ ). Coll 1971 by O R Ortiz-Troncoso and subm by A Laming-Emperaire. Comment: Lauta dates are quoted by Rapaire and Hugues, 1977, in connection with Lancha Packewaia series, Tierra del Fuego, on opposite shore of Beagle Channel (Ortiz-Troncoso, 1977-78; 1978).

Gif-2729. Lauta, Navarino I., Chile, Pit 3

$2780 \pm 110$

Shells coll in similar conditions to those of Gif-2728 from another site at Seno del Lauta, subm by A Laming-Emperaire. Comment: date represents relatively early occupation phase of that coast.

Gif-2927. Bahia Buena, Chilean Patagonia

$5210 \pm 110$

Charcoal from oldest occupation layer of shell midden overlying old shoreline, 10.5 to $12 \mathrm{~m}$ above msl, $400 \mathrm{~m}$ from Magellan Strait $\left(53^{\circ} 36^{\prime} \mathrm{S}\right.$, $\left.70^{\circ} 59^{\prime} \mathrm{W}\right)$. Coll 1973 by $\mathrm{O} \mathrm{R}$ Ortiz-Troncoso and subm by A LamingEmperaire. Comment: stratigraphic sequence of site indicates lower cultural layer (II) overlying clayey substratum of morainic origin (or Layer I), two middle sub-layers (III \& IIIb), and upper or most recent layer (IV). Components of deposits are shells, bones (mammals and birds), fish remains, sea urchin remains, sand, gravel, stones, ash, and charcoal. Most important characteristic of lithic industry is large percentage of obsidian. Typical artifacts of bone industry are harpoon heads with bilaterally lobed tangs, multibarbed harpoon heads, and other tools with incised decorations. Cultural assemblage related to that from Englefield I 
site (Emperaire and Laming, 1961); Punta Santa Ana site (Ortiz-Troncoso, 1975; 1977-78; 1978; 1979); and Lancha Packewaia and Tunel (Orquera et al, 1978, R, 1977, v 19, p 49-61). Date confirmed by GrN-7613 and -7614: $5770 \pm 110$ and $5895 \pm 65 \mathrm{BP}$.

\section{Gif-2928. Punta Santa Ana, Chilean Patagonia}

Shells from oldest occupation layer of shell midden overlying clayey substratum of morainic origin, $12 \mathrm{~m}$ above $\mathrm{msl}\left(53^{\circ} 37^{\prime} \mathrm{S}, 70^{\circ} 58^{\prime} \mathrm{W}\right)$. Coll 1972 by $\mathrm{O} R$ Ortiz-Troncoso and subm by A Laming-Emperaire. Comment: stratigraphic sequence and components of deposits are quite similar to those of neighboring Bahia Buena site, Gif-2927. Another date for this layer is GrN-7612: $6410 \pm 70 \mathrm{BP}$.

\section{REFERENCES}

Battistini, R, Delibrias, G, and Laborel, J, 1976, Datation des niveaux holocènes à Madagascar: Soc Géol France Compte rendu somm, fasc 6, p 284-285.

Briard, J, 1972, Les tumulus de Kervin en Poullan: Soc Archéol Bull Finistère, v 98, p $21-38$.

Briard, J, Bourhis, J, Le Provost, F, and Onnée, Y, 1977, Un tumulus du bronze ancien avec maison funéraire à Saint-Jude, Bourbriac, Côtes du Nord: Soc Préhist Fr Bull, v 74, p 622-664.

Briard, J and Gouletquer, P L, 1972, Découverte d'une tombe de l'âge du Bronze à Kerno en Ploudaniel, Finistère: Annales Bretagne, v 79, p 49-60.

Briard, J and Penziat, J, 1972, Le tumulus de Penguilly en Meilars, Finistìre: Annales Bretagne, v 79, no. 1, p 63-72.

Charon, M, Ortlieb, L, and Petit-Maire, N, 1973, Occupation humaine holocène de la région du Cap Juby: Soc Anthropol Paris Bull et Mém, v 10, ser 12, p 379-412.

Courtin, J, 1973, Datation $\mathrm{C}^{14}$ du Mésolithique de la Baume Fontbrégoua à Salernes, Var: Soc Préhist Fr Bull, v 70, no. 4, p 99-100.

Contenson, H, de, 1973, Chronologie absolue de Tell Aswad (Damascène, Syrie): Soc Prćhist Fr Bull, v 70, CRSM, no. 8.

1976. Nouvelles données sur le Néolithique précéramique dans la région de Damas (Syrie) d'après les fouilles de Ghoraifé en 1974: Soc Préhist Fr Bull, v 73, ORSM no. 3.

Delibrias, G, Giresse, P, and Kouyoumontzakis, G, 1973, Géochronologie des divers stades de la transgression holocène au large du Congo: Acad Sci [Paris] Comptes rendus, v 276, p 1389-1391.

Delibrias, G, Guillier, M T, and Labeyrie, J, 1964, Saclay natural radiocarbon measurements I: Radiocarbon, v 6, p 233-250.

320. 1972, Gif natural radiocarbon measurements VII: Radiocarbon, v 14, p 280-

320.51974 , Gif natural radiocarbon measurements VII: Radiocarbon, v 16, p 15-94.

Delibrias, $G$ and Le Roux, C T, 1975, Un exemple d'application des datations radiocarbone à l'interprétation d'une stratigraphie complexe: la fouille des ateliers de Plussulien, Côtes du Nord: Soc Préhist Fr Bull, v 72, p 78-82.

Delibrias, G, Marinclli, G, and Sticlies, L, 1974, Spreading rate of the Asal Rift: a geological approach: Internatl symposium on Afar region and related rift problems, Proc: Bad Bergzabern, West Germany, v 1, p 212-221.

Delibrias, $G$ and Morzadec-Kerfourn, M T, 1975, Evolution du Marais de Dol de Bretagne au Flandrien, Ille et Vilaine, France: Assoc Fr Etude Quaternaire Bull, v $2, \mathrm{p} 59-70$.

Delibrias, G, Ortlieb, L, and Petit-Maire, N, 1976, New ${ }^{14} \mathrm{C}$ data for the Atlantic Sahara (Holocène). Tentative paleoclimatic interpretation: Human evol Jour, v 5, p 535-546.

Delibrias, G and Roche, J, 1976, Datations absolues de l'Epipaléolithique marocain: Archéol Marocaine Bull, v 10, p $11-24$.

Emperaire, J and Laming, A, 1961, Les gisements des îles Englefield et Vivian dans la mer d'Otway, Patagonie australe: Soc Américanistes Jour, ns, v 50, p 7-75.

Evin, J, Marien, G, and Pachiaudi, Ch, 1978, Lyon natural radiocarbon measurements VIII: Radiocarbon, v 20, p 19-57.

Faure, H, Hoang, C T, and Lalou, C, 1980, Datations ${ }^{230} \mathrm{Th} /{ }^{234} \mathrm{U}$ des calcaires coralliens et mouvements verticaux à Djibouti: Soc Géol France Bull, v 22, no. 7, p 959-962. 
Fontaine, H and Delibrias, G, 1974, Niveaux marins pendant le Quaternaire au VietNam: Archéol Géol Viet-Nam, Saīgon, v 17, p 35-44.

Gabet, C, 1974, Le banc de tourbe sur l'estran de la baie de Perroche (Ile d'Oléron): Soc Archéol Hist Charente Maritime Rec, v 25, p 297-306.

1978, Note sur les marais de Saint-Augustin et d'Arvert (Charente Maritime): Poitiers, Norois, v 100, p 609-614.

Germain, P, 1975, Contribution à la connaissance du Quaternaire récent du littoral Dahomien: Assoc Sénégal Quaternaire Afr, liaison Sénégal Bull, v 44, p 33-45.

Giot, P R and Cogné, J, 1951, La Nécropole de Saint-Urnel en Plomeur, fouilles de 1946-1950: Gallia, v9, p 1-19.

Giot, P R and Monnier, J L, 1977, Le cimetic̀re des anciens Bretons de Saint-Urnel ou Saint-Saturnin en Plomeur, Finistère: Gallia, v 35, p 141-171.

1978, Les oratoires des anciens Bretons de Saint-Urnel ou Saint-Saturnin en Plomeur, Finistère: Archćol Médiévale, v 8, p 55-93.

Giresse, P, Kouyoumontzakis, G, and Delibrias G, 1976, La transgression fini-holocène en Angola, aspects chronologique, eustatique, paléoclimatique et épirogénique: Acad Sci [Paris] Comptes rendus, v 283, p 1157-1160.

Giresse, P and Lautridou, J P, 1973, Les formations quaternaires du littoral du Golfe Normand-Breton entre Coutainville et Avranches: Assoc Fr Quaternaire Bull, v 2, p 2-89.

Guilcher, A, 1974, Coral reefs of the New Hebrides Melanesia, with particular reference to open-sea, not fringing reefs: Internatl Coral Reef symposium, 2nd, Proc: Great Barrier Reef Comm, Brisbane, p 523-535.

Guillien, Y, 1975, Caractère cyclothémique et datation absolue intragréseux de Sonneville, Charente: Cong natl Soc Savantes, 99th, Actes: Besançon, v 1, p 65-70.

Guillien, Y and Puisségur, J J, 1969, Géomorphologie et malacologie de quelques dépôts de pente charentais: Acad Sci [Paris] Comptes rendus, v 268, p 24-27.

Huault, M F and Auffret, J P, 1975, Etudes de dépôts quaternaires sous-marins en Manche orientale: Assoc Fr Quaternaire Bull, v 1, p 43-49.

Ichon, A, 1977, Les sculptures de La Lagunita, El Quiché, Guatemala, in Piedra Santa, ed, Guatemala, 73 p.

Joussaume, R, 1971, Les monuments mégalithiques du Harrar, Ethiopie: L'Anthropologie, v 15, no. 3-4, p 177-190.

Laborel, J and Delibrias, G, 1976, Niveaux marins récents à vermetidae du littoral Ouest Africain, comparaison avec les niveaux homologues des Côtes du Brásil: Assoc Sénégal Quaternaire Afr, liaison Sénégal Bull, v 47, p 97-110.

Langouët, L and Meury, J L, 1973, La machinerie en bois du Haut Empire retrouvée à Alet: Annales Bretagne, v 80, no. 1, p 163-184.

Lecornec, $\mathrm{J}, 1973$, Le site à enclos de Kerlande à Brandivy, Morbihan: Annales Bretagne, v 80, p 61-70.

Léger, M, 1965, Les terrasses du Danube de Regensburg à Pleinting: Assoc Fr Etude Quaternaire Bull, v 3, p 153-164.

Mariette, H, 1959, Civilisation des Gobclets de la région boulonnaise: Cong Préhist, 16 th sess, p 853-859.

Mary, G, Medus, J, and Delibrias, G, 1975, Le Quaternaire de la côte Asturienne, Espagne: Assoc Fr Etude Quaternaire Bull, v 1, p 13-23.

Morand, F, 1971, Contribution à l'étude de la formation des marais et tourbic̀res de Cessières et Montbavin, Aisne: Assoc Géol Fr Bull, no. 387-388, p 97-116.

Orquera, L A, Sala, A E, Piana, E L, and Tapia, A H, 1978, Lancha Packewaia: Arqueologia de los canales fueguinos: Buenos Aires, Argentina, Ed Huemul, S A.

Ortiz-Troncoso, C R, 1975, Los yacimientos de Punta Santa Ana y Bahia Puena, Patagonia austral. Excavationes y fechados radiocarbonicos: Inst Patagonia Anales, v 6, p 93-122.

1977-78, Nuevas datationes radiocarbonicas para Chile austral, Patagonia y Tierra des Fuegos: Mus Arqueol Serena Bol, no. 16, p 244-250.

1978, Nuevo fechado radiocarbonico para la isla Englefield, seno Otway, Patagonia austral: Soc Argentina Antropol Rel, ns, v 12, p 243-244.

1979, Punta Santa Ana et Bahia Buena: deux gisements sur une ancienne ligne de rivage dans le détroit de Magellan: Soc Américanistes Jour, ns, v 66, p 133-204.

Paradis, G, ms, 1975, Recherches sur le Quaternaire récent du sud de la République du Bénin, ex Dahomey: Etude de thanatocénoses de mollusques: 'Thesis, 3rd cycle, Univ Paris Sud, Orsay.

1978, Interprétation paléoécologique et paléogéographique des Taphocénoses de l'Holocène récent du sud Bénin, à partir de la répartition actuelle des mollusques littoraux et lagunaires d'Afrique occidentale: Géobios, v 2, no. 6, p 867-891. 
Petit-Maire, N, 1979, Le Sahara atlantique à l'Holocène. Peuplement et ćcologie: Centre recherche anthrop préhist ethnol mém, v 28, Algiers.

Puyfaucher, A and Amat, J P, 1972, Fragments d'os et silex taillés découverts dans la plaine de Chanfroy: Inf Géol Bass Paris, v 32, p 53-55.

Puyfaucher, A and Collin, F, 1971, Les grèves des plaines de Chanfroy, de la Feuillardière et du Cul du Chaudron, Forêt de Fontainebleau: Assoc Fr Etude Quaternaire Bull, v 4, p 241-249.

Rapaire, J L and Hugues, G, 1977, Monaco radiocarbon measurements V: Rádiocarbon, v 19, p 49-61.

Richard, P, 1971, Two pollen diagrams from the Québec city area, Canada: Pollen et Spores, v 13 , no. 4 , p 523-559.

Roche, J, 1976, Cadre chronologique de l'Epipalćolithique marocain, chronologie et synchronisme dans la Préhistoire circum-méditerranéenne: Cong UISIPP, 9th, Nice, Colloque II, p 153-167.

Roche, J and Texier, J P, 1976, Découverte de restes humains dans un niveau atérien supérieur de la grotte des Contrebandiers, à Tamara, Maroc: Acad Sci [Paris] Comptes rendus, v 282, ser D, p 45-47.

Sacchi, D, 1972, Datage ${ }^{14} \mathrm{C}$ d'un gisement mésolithique des Corbières, La Cauna d'Arques, Aude: Soc Préhist Fr Bull, v 69, no. 8, p 7.

Stieljes, L, 1973, Evolution tectonique récente du rift d'Asal TFAI: Rev Géog Phys Géol Dynamique, v 15, no. 4, p 425-436.

Ters, M, 1973, Les variations du niveau marin depuis 10.000 ans le long du littoral atlantique français: Le Quaternaire géodynamique, stratigraphie et environment: Cong internatl INQUA, 9th, Christchurch, New Zealand.

Thévenin, A G, 1970, Lingolsheim, informations archéologiques: Gallia Préhist, v 13, fasc $2, \mathrm{p} 397$.

Thévenin, A G and Sainty, J, 1972, Une nouvelle stratigraphie du Post Glaciaire: l'abri du Mannlefelsen I, à Oberlag, Haut Rhin: Soc Préhist Fr Bull, v 69, CRSM no. 1,

p 6-7. 1972, L'abri de Rochedane à Villars-sous-Dempjoux, Doubs: Rev Archéol Est, v 23, fasc 1, p 7-22.

Verger, F, 1971, Présentation taxonomique des formes des marais maritimes tempérés: Rev Géog Phys Géol Dynamique, v 13, no. 2, p 163-171. 